\title{
Measurement of Circumstellar Disk Sizes in the Upper Scorpius OB Association with ALMA
}

\author{
Scott A. Barenfeld ${ }^{1}$ (D), John M. Carpenter ${ }^{2}$ (D), Anneila I. Sargent ${ }^{1}$, Andrea Isella ${ }^{3}$ (D), and Luca Ricci ${ }^{3}$ \\ ${ }^{1}$ California Institute of Technology, Department of Astronomy, MC 249-17, Pasadena, CA 91125, USA \\ ${ }^{2}$ Joint ALMA Observatory, Av. Alonso de Córdova 3107, Vitacura, Santiago, Chile \\ ${ }^{3}$ Department of Physics and Astronomy, Rice University, 6100 Main Street, Houston, TX 77005, USA \\ Received 2017 June 30; revised 2017 October 27; accepted 2017 November 4; published 2017 December 15
}

\begin{abstract}
We present detailed modeling of the spatial distributions of gas and dust in 57 circumstellar disks in the Upper Scorpius OB Association observed with ALMA at submillimeter wavelengths. We fit power-law models to the dust surface density and $\mathrm{CO} J=3-2$ surface brightness to measure the radial extent of dust and gas in these disks. We found that these disks are extremely compact: the 25 highest signal-to-noise disks have a median dust outer radius of $21 \mathrm{au}$, assuming an $R^{-1}$ dust surface density profile. Our lack of $\mathrm{CO}$ detections in the majority of our sample is consistent with these small disk sizes assuming the dust and CO share the same spatial distribution. Of seven disks in our sample with well-constrained dust and $\mathrm{CO}$ radii, four appear to be more extended in CO, although this may simply be due to the higher optical depth of the CO. Comparison of the Upper Sco results with recent analyses of disks in Taurus, Ophiuchus, and Lupus suggests that the dust disks in Upper Sco may be approximately three times smaller in size than their younger counterparts, although we caution that a more uniform analysis of the data across all regions is needed. We discuss the implications of these results for disk evolution.
\end{abstract}

Key words: open clusters and associations: individual (Upper Scorpius OB1) - planetary systems - protoplanetary disks - stars: pre-main sequence

Supporting material: extended figures

\section{Introduction}

The past two decades have seen tremendous progress in our understanding of protoplanetary disks (see the recent reviews by Williams \& Cieza 2011; Alexander et al. 2014; Dutrey et al. 2014; Espaillat et al. 2014; Pontoppidan et al. 2014; Testi et al. 2014; Andrews 2015). Submillimeter interferometry has played a crucial role, allowing the gas and dust throughout disks to be studied at high spatial resolution. At submillimeter wavelengths, the dust continuum emission from disks is mostly optically thin, making it possible to measure dust masses and surface densities. Additionally, a number of molecular species present in disks have rotational lines observable in the submillimeter that can be used to study disk temperature, chemistry, kinematics, and mass.

Early submillimeter observations with interferometers focused on young, bright disks, revealing objects that were hundreds of astronomical units in size with masses of a few percent of their host stars (e.g., Kitamura et al. 2002; Andrews \& Williams 2007; Isella et al. 2007, 2009, 2010a, 2010b; Andrews et al. 2009; Guilloteau et al. 2011). Subsequent observations of fainter disks indicated that smaller sizes and masses may be more typical (Andrews et al. 2013; Piétu et al. 2014; Testi et al. 2016; Hendler et al. 2017; Tazzari et al. 2017). More recently, a number of studies targeted older protoplanetary disks, which are crucial to our understanding of how disks evolve and dissipate. Pre-ALMA surveys of IC348 (Lee et al. 2011), the Upper Scorpius OB Association (hereafter Upper Sco, Mathews et al. 2012), and $\sigma$ Orionis (Williams et al. 2013), revealed a dearth of evolved disks comparable to the brightest objects in younger regions, suggesting that older disks are intrinsically fainter than their younger counterparts.

With ALMA, it is possible to conduct large surveys of disks at an unprecedented level of sensitivity, revealing the properties of unbiased samples within individual stellar populations. Thus, Ansdell et al. (2016) surveyed the 1-3 Myr old Lupus star-forming region and, from separate measurements of dust and gas masses in 89 disks, found evidence that $\mathrm{CO}$ is depleted relative to dust compared to interstellar medium (ISM) values (see also Miotello et al. 2017). Eisner et al. (2016) and Ansdell et al. (2017) found evidence for similar depletion in the $<1 \mathrm{Myr}$ old Orion Nebula cluster and the 3-5 Myr old $\sigma$ Orionis region. In a survey of 93 disks in the 2-3 Myr old Chamaeleon star-forming region, Pascucci et al. (2016) found a relationship between disk dust mass and stellar mass consistent with other 1-3 Myr old regions, but with a shallower slope than is seen for older disks.

Crucially, the sensitivity of ALMA enables disk surveys to be extended to more evolved stellar populations (e.g., Carpenter et al. 2014; Hardy et al. 2015; van der Plas et al. 2016; Barenfeld et al. 2016, hereafter Paper I). In particular, Upper Sco provides an ideal target for such studies. The 5-11 Myr age of this association (Preibisch et al. 2002; Pecaut et al. 2012) implies that its protoplanetary disks are in the last stage of evolution before dissipation (Hernández et al. 2008). Based on ALMA observations of 20 disk-bearing stars in Upper Sco, Carpenter et al. (2014) found tentative evidence that disk dust masses are lower than in the younger Taurus star-forming region. Paper I expanded this sample to include ALMA observations of 106 Upper Sco disks and found that the dust masses are on average a factor of 4.5 lower than those in Taurus with high statistical significance. Of the 58 sources detected in the continuum in this survey, the majority were not spatially resolved, implying dust disk radii of a few tens of astronomical units or less. Only 26 sources were detected in $\mathrm{CO}$, suggesting that the $\mathrm{CO}$ is also confined to a compact emitting area or is heavily depleted relative to the dust. 
Here we present a more detailed study of the gas and dust for the disks in the Paper I sample and build on our previous results by measuring disk sizes, modeling $\mathrm{CO}$ emission, and determining the relative distributions of gas and dust. In Section 2, we describe our disk sample and ALMA observations. In Section 3, we detail our methodology for modeling the continuum emission, while our modeling of the $\mathrm{CO}$ emission is described in Section 4. We then discuss the implications of the gas and dust properties of these disks in Section 5. Our conclusions are summarized in Section 6.

\section{Sample and Observations}

Our stellar sample for the current work is a subset of the parent sample described in detail in Paper I. Briefly, our parent sample consisted of 106 stars in Upper Sco with spectral types between G2 and M5 (inclusive) and included all 100 candidate G2-M4.75 disk-host stars in Upper Sco identified by Luhman \& Mamajek (2012) using Spitzer and WISE observations, as well as six M5 disk-host candidates from Carpenter et al. (2006) found using Spitzer observations.

In this work, we analyzed 57 sources detected in Paper I, listed in Tables 1 and 2. Of these sources, 21 were detected in both the $0.88 \mathrm{~mm}$ continuum and the $\mathrm{CO} J=3-2$ line at $345.79599 \mathrm{GHz}, 34$ were only detected in the continuum, and two were detected only in CO. Five of the sources analyzed are classified as debris/evolved transitional disks by Luhman \& Mamajek (2012). We consider the remaining sources to be primordial disks (see Paper I).

Three extremely bright continuum sources were identified in Paper I, 2MASS J15583692-2257153, 2MASS J160421652130284, and 2MASS J16113134-1838259, which have continuum flux densities of $174.92 \pm 0.27 \mathrm{mJy}, 218.76 \pm$ $0.81 \mathrm{mJy}$, and $903.56 \pm 0.85 \mathrm{mJy}$ at $0.88 \mathrm{~mm}$, respectively. 2MASS J15583692-2257153 exhibits an azimuthal asymmetry in the continuum, while 2MASS J16042165-2130284 shows the large inner cavity of a transitional disk. 2MASS J16113134-1838259 is more than 20 times brighter in the continuum than any of the sources we are including in this paper and exhibits possible disk winds and tidal interactions with a stellar companion (Salyk et al. 2014). Since these systems are not representative of typical disks in Upper Sco, we excluded them from the present analysis and focused instead on understanding the broader population of ordinary disks. Zhang et al. (2014) presented a detailed analysis of 2MASS J16042165-2130284 (see also Mayama et al. 2012; Pinilla et al. 2015; van der Marel et al. 2015; Dong et al. 2017).

The ALMA observations were obtained in Cycle 0 and Cycle 2 using the $12 \mathrm{~m}$ array (see Paper I). All observations used band 7 , with a mean frequency of $340.7 \mathrm{GHz}$ for Cycle 0 and $341.1 \mathrm{GHz}$ for Cycle $2(0.88 \mathrm{~mm})$ and a total bandwidth of $7.5 \mathrm{GHz}$. One spectral window was configured with channel widths of $0.488 \mathrm{MHz}\left(0.429 \mathrm{~km} \mathrm{~s}^{-1}\right.$, the spectral resolution is twice the channel width) to observe the CO $J=3-2$ line at $345.79599 \mathrm{GHz}$. The observations had angular resolution between 0. " 35 and 0 !" 73 with a median of $0 . ! 37$ and a continuum rms ranging from $0.13 \mathrm{mJy} /$ beam to $0.26 \mathrm{mJy} /$ beam, with a median of $0.15 \mathrm{mJy} /$ beam.

\section{Continuum Modeling}

Our goal in modeling the continuum data was to determine the radial extent of the dust for the 55 continuum-detected disks in our sample. To accomplish this, we compared our observed visibilities to the synthetic visibilities of a model disk, deriving the model visibilities from an assumed dust density distribution in a self-consistent way.

For our model disk, we parameterized the dust surface density as a function of radius using a truncated power law:

$$
\Sigma(R)=\Sigma_{0}\left(\frac{R}{10 \mathrm{au}}\right)^{-1},
$$

for $R$ between the dust inner and outer radii, $R_{\mathrm{in}}$ and $R_{\mathrm{dust}}$, with $\Sigma=0$ outside of this range. We fixed $R_{\text {in }}$ at the values found by the SED fitting of Mathews et al. (2013) for the 24 sources we share with their survey. For our remaining sources, we set $R_{\text {in }}$ to be equal to the dust sublimation radius, calculated based on the stellar luminosities from Paper I. The choice of $R_{\text {in }}$ does not impact our results.

The continuum signal-to-noise ratio for the majority of our sample is too low to simultaneously constrain the dust outer radius and the slope of the surface density power law. We therefore adopted a fixed $R^{-1}$ parameterization for the surface density, which is fairly typical for disks (e.g., Kitamura et al. 2002; Andrews \& Williams 2007; Isella et al. 2007, 2010a; Guilloteau et al. 2011). Assuming $\Sigma \propto R^{-0.5}$ and $\Sigma \propto R^{-1.5}$ power laws resulted in slightly smaller or larger disk sizes, respectively, but did not qualitatively impact our results or conclusions.

Some authors (e.g., Hughes et al. 2008, 2010; Andrews et al. 2009, 2010a, 2010b, 2011, 2012; Isella et al. 2009, 2010a, 2010b; Guilloteau et al. 2011) have parameterized the disk surface density using the self-similar solution for a viscously evolving disk, which can be approximated as a power law with an exponential tail. However, given the evolutionary state of the Upper Sco disks, it is not clear that this description is appropriate. Other effects, such as the inward radial migration of dust grains (e.g., Weidenschilling 1977), may also change the surface density profile. Birnstiel \& Andrews (2014) modeled dust transport in a viscously evolving disk and found that grain migration results in a dust surface density welldescribed by a power law with a sharp outer edge. We therefore adopted a power-law surface density for our analysis. Broken power-law models have also been used to model dust surface density (e.g., Hogerheijde et al. 2016) but given the low signalto-noise of the majority of our detections, we opted for a single power-law model with fewer free parameters.

The vertical disk structure was parameterized using the commonly assumed Gaussian vertical density structure of an isothermal disk (e.g., Isella et al. 2007):

$$
\rho(R, z)=\frac{\Sigma(R)}{h \sqrt{2 \pi}} \exp \left(\frac{-z^{2}}{2 h^{2}}\right) .
$$

The disk scale height, $h$, was assumed to be a power-law function of radius

$$
h=h_{0}\left(\frac{R}{100 \mathrm{au}}\right)^{p} .
$$

We allowed $h_{0}$ and $p$ to vary with priors based on the SED fits of Mathews et al. (2013). Our choice of these priors does not affect our conclusions. Thus, if a disk was included in the Mathews et al. (2013) sample, we used their best-fit values for $h_{0}$ and $p$ with uncertainties of 1 au and 0.05 to create normally 
Table 1

Continuum Fitting Results

\begin{tabular}{|c|c|c|c|c|c|c|}
\hline Source & $\log \frac{\Sigma_{0}}{\mathrm{~g} \mathrm{~cm}^{-2}}$ & $R_{\text {dust }}(\mathrm{au})$ & $p$ & $h_{0}(\mathrm{au})$ & Inclination (deg) & Position Angle (deg) \\
\hline 2MASS J15354856-2958551 & $-2.45(-0.19,+0.24)$ & $14(-10,+18)$ & $1.12(-0.04,+0.06)$ & $3(-3,+7)$ & $46(-34,+17)$ & $25(-25,+121)$ \\
\hline 2MASS J15514032-2146103 & $-2.78(-0.25,+0.29)$ & $13(-10,+35)$ & $1.12(-0.04,+0.06)$ & $8(-8,+1)$ & $84(-59,+4)$ & $130(-95,+46)$ \\
\hline 2MASS J15530132-2114135 & $-1.41(-0.20,+0.32)$ & $8(-2,+5)$ & $1.12(-0.04,+0.05)$ & $8(-4,+6)$ & $47(-40,+14)$ & $28(-25,+117)$ \\
\hline 2MASS J15534211-2049282 & $-2.01(-0.61,+1.07)$ & $45(-7,+21)$ & $1.13(-0.06,+0.04)$ & $1(-1,+1)$ & $89(-2,+1)$ & $73(-6,+5)$ \\
\hline 2MASS J15582981-2310077 & $-1.58(-0.14,+0.21)$ & $13(-3,+10)$ & $1.13(-0.07,+0.04)$ & $6(-1,+1)$ & $32(-21,+18)$ & $47(-32,+115)$ \\
\hline 2MASS J16001844-2230114 & $-1.98(-0.13,+0.16)$ & $30(-9,+5)$ & $1.13(-0.05,+0.05)$ & $5(-3,+6)$ & $45(-21,+35)$ & $6(-48,+36)$ \\
\hline 2MASS J16014086-2258103 & $-2.12(-0.17,+0.14)$ & $36(-9,+9)$ & $1.12(-0.06,+0.05)$ & $8(-3,+10)$ & $74(-31,+10)$ & $26(-23,+22)$ \\
\hline 2MASS J16014157-2111380 & $-2.60(-0.36,+0.38)$ & $9(-9,+18)$ & $1.13(-0.05,+0.05)$ & $3(-3,+6)$ & $80(-58,+5)$ & $160(-105,+20)$ \\
\hline 2MASS J16020757-2257467 & $-2.17(-0.12,+0.21)$ & $47(-7,+8)$ & $1.12(-0.03,+0.07)$ & $1(-1,+16)$ & $57(-19,+14)$ & $80(-15,+17)$ \\
\hline 2MASS J16024152-2138245 & $-1.37(-0.17,+0.14)$ & $24(-3,+3)$ & $1.13(-0.04,+0.05)$ & $8(-3,+7)$ & $41(-21,+14)$ & $63(-21,+28)$ \\
\hline 2MASS J16030161-2207523 & $-1.86(-0.19,+0.24)$ & $19(-8,+7)$ & $1.13(-0.04,+0.05)$ & $7(-4,+8)$ & $52(-42,+22)$ & $62(-50,+46)$ \\
\hline 2MASS J16032225-2413111 & $-2.20(-0.19,+0.30)$ & $15(-11,+13)$ & $1.12(-0.04,+0.04)$ & $6(-3,+12)$ & $64(-36,+16)$ & $72(-43,+56)$ \\
\hline 2MASS J16035767-2031055 & $-2.51(-0.10,+0.10)$ & $115(-46,+88)$ & $1.11(-0.05,+0.05)$ & $10(-1,+1)$ & $69(-27,+21)$ & $5(-26,+22)$ \\
\hline 2MASS J16035793-1942108 & $-2.75(-0.09,+0.10)$ & $173(-60,+46)$ & $1.14(-0.05,+0.05)$ & $9(-1,+1)$ & $56(-34,+14)$ & $42(-42,+34)$ \\
\hline 2MASS J16041740-1942287 & $-2.62(-0.24,+0.34)$ & $9(-8,+14)$ & $1.12(-0.05,+0.05)$ & $4(-3,+6)$ & $80(-50,+7)$ & $100(-79,+60)$ \\
\hline 2MASS J16043916-1942459 & $-2.53(-1.36,+1.10)$ & $46(-42,+21)$ & $1.13(-0.05,+0.05)$ & $4(-4,+7)$ & $77(-54,+9)$ & $22(-18,+123)$ \\
\hline 2MASS J16052556-2035397 & $-2.46(-0.19,+0.28)$ & $16(-12,+38)$ & $1.21(-0.05,+0.05)$ & $3(-1,+1)$ & $74(-23,+16)$ & $91(-68,+72)$ \\
\hline 2MASS J16054540-2023088 & $-1.72(-0.08,+0.07)$ & $19(-2,+5)$ & $1.22(-0.04,+0.06)$ & $9(-1,+1)$ & $67(-29,+9)$ & $10(-10,+36)$ \\
\hline 2MASS J16062196-1928445 & $-2.77(-0.13,+0.13)$ & $46(-16,+32)$ & $1.13(-0.05,+0.05)$ & $13(-1,+1)$ & $85(-68,+5)$ & $121(-52,+39)$ \\
\hline 2MASS J16062277-2011243 & $-2.99(-0.34,+0.33)$ & $9(-8,+33)$ & $1.22(-0.05,+0.05)$ & $6(-1,+1)$ & $85(-50,+5)$ & $161(-127,+13)$ \\
\hline 2MASS J16063539-2516510 & $-2.28(-0.20,+0.16)$ & $43(-19,+17)$ & $1.14(-0.06,+0.04)$ & $8(-3,+8)$ & $74(-43,+13)$ & $11(-11,+70)$ \\
\hline 2MASS J16064102-2455489 & $-1.96(-0.12,+0.24)$ & $29(-8,+8)$ & $1.13(-0.05,+0.05)$ & $6(-4,+8)$ & $40(-36,+14)$ & $81(-41,+48)$ \\
\hline 2MASS J16064385-1908056 & $-3.04(-0.20,+0.23)$ & $17(-16,+62)$ & $1.19(-0.05,+0.05)$ & $3(-1,+1)$ & $48(-39,+38)$ & $81(-36,+81)$ \\
\hline 2MASS J16072625-2432079 & $-1.50(-0.13,+0.20)$ & $29(-2,+2)$ & $1.13(-0.05,+0.04)$ & $6(-4,+4)$ & $43(-17,+10)$ & $2(-14,+19)$ \\
\hline 2MASS J16072747-2059442 & $-2.19(-0.17,+0.26)$ & $11(-5,+9)$ & $1.12(-0.04,+0.06)$ & $4(-4,+6)$ & $68(-49,+10)$ & $20(-20,+106)$ \\
\hline 2MASS J16073939-1917472 & $-3.10(-0.34,+0.33)$ & $9(-9,+73)$ & $1.13(-0.05,+0.05)$ & $2(-1,+8)$ & $83(-75,+7)$ & $148(-117,+31)$ \\
\hline 2MASS J16075796-2040087 & $-0.64(-0.22,+0.13)$ & $11(-1,+1)$ & $1.18(-0.04,+0.04)$ & $18(-4,+1)$ & $47(-14,+8)$ & $0(-14,+15)$ \\
\hline 2MASS J16081566-2222199 & $-2.89(-0.16,+0.19)$ & $80(-41,+59)$ & $1.13(-0.05,+0.05)$ & $8(-6,+5)$ & $86(-26,+4)$ & $173(-18,+24)$ \\
\hline 2MASS J16082324-1930009 & $-1.10(-0.15,+0.18)$ & $65(-5,+5)$ & $1.16(-0.07,+0.04)$ & $8(-1,+1)$ & $74(-4,+5)$ & $123(-2,+3)$ \\
\hline 2MASS J16082751-1949047 & $-2.72(-0.19,+0.21)$ & $44(-35,+21)$ & $1.11(-0.05,+0.05)$ & $2(-1,+1)$ & $41(-34,+34)$ & $17(-11,+132)$ \\
\hline 2MASS J16090002-1908368 & $-2.57(-0.14,+0.31)$ & $9(-7,+18)$ & $1.24(-0.05,+0.06)$ & $19(-1,+1)$ & $63(-45,+18)$ & $84(-38,+81)$ \\
\hline 2MASS J16090075-1908526 & $-1.27(-0.06,+0.07)$ & $58(-4,+5)$ & $1.13(-0.05,+0.05)$ & $6(-1,+1)$ & $56(-5,+5)$ & $149(-9,+9)$ \\
\hline 2MASS J16093558-1828232 & $-2.87(-0.31,+0.36)$ & $7(-7,+28)$ & $1.13(-0.05,+0.05)$ & $3(-3,+6)$ & $83(-59,+6)$ & $104(-81,+40)$ \\
\hline 2MASS J16094098-2217594 & $-3.58(-0.35,+0.36)$ & $12(-10,+62)$ & $1.13(-0.05,+0.05)$ & $1(-1,+13)$ & $82(-61,+6)$ & $74(-53,+65)$ \\
\hline 2MASS J16095361-1754474 & $-2.76(-0.21,+0.31)$ & $6(-6,+28)$ & $1.18(-0.05,+0.05)$ & $9(-1,+1)$ & $86(-60,+4)$ & $154(-131,+16)$ \\
\hline 2MASS J16095441-1906551 & $-3.11(-0.58,+0.52)$ & $7(-7,+41)$ & $1.13(-0.05,+0.05)$ & $2(-2,+7)$ & $83(-72,+5)$ & $177(-42,+48)$ \\
\hline 2MASS J16095933-1800090 & $-3.56(-0.30,+0.34)$ & $8(-6,+63)$ & $1.14(-0.04,+0.06)$ & $16(-1,+1)$ & $86(-66,+4)$ & $105(-64,+59)$ \\
\hline 2MASS J16102857-1904469 & $-3.02(-0.44,+0.40)$ & $9(-9,+28)$ & $1.14(-0.06,+0.05)$ & $2(-2,+12)$ & $84(-51,+6)$ & $98(-74,+43)$ \\
\hline 2MASS J16104636-1840598 & $-2.13(-0.25,+0.36)$ & $10(-8,+15)$ & $1.12(-0.04,+0.06)$ & $8(-5,+8)$ & $71(-63,+8)$ & $84(-38,+78)$ \\
\hline 2MASS J16111330-2019029 & $-1.69(-0.27,+0.15)$ & $8(-2,+8)$ & $1.14(-0.06,+0.04)$ & $6(-3,+12)$ & $17(-13,+40)$ & $141(-78,+35)$ \\
\hline 2MASS J16115091-2012098 & $-2.94(-0.25,+0.24)$ & $95(-53,+6)$ & $1.13(-0.04,+0.06)$ & $1(-1,+8)$ & $86(-42,+4)$ & $144(-44,+32)$ \\
\hline 2MASS J16122737-2009596 & $-2.98(-0.30,+0.35)$ & $86(-43,+15)$ & $1.13(-0.05,+0.05)$ & $1(-1,+8)$ & $26(-14,+50)$ & $159(-112,+18)$ \\
\hline 2MASS J16123916-1859284 & $-2.21(-0.10,+0.20)$ & $48(-7,+8)$ & $1.12(-0.05,+0.05)$ & $8(-5,+8)$ & $51(-36,+14)$ & $46(-27,+22)$ \\
\hline 2MASS J16133650-2503473 & $-2.82(-0.26,+0.26)$ & $45(-33,+48)$ & $1.14(-0.06,+0.04)$ & $4(-2,+12)$ & $86(-52,+4)$ & $23(-29,+105)$ \\
\hline 2MASS J16135434-2320342 & $-1.18(-0.59,+0.86)$ & $10(-3,+3)$ & $1.14(-0.05,+0.05)$ & $6(-5,+5)$ & $52(-44,+14)$ & $75(-49,+52)$ \\
\hline 2MASS J16135434-2320342B & $-1.60(-0.17,+0.25)$ & $13(-3,+5)$ & $1.14(-0.05,+0.04)$ & $6(-1,+12)$ & $40(-34,+10)$ & $154(-88,+29)$ \\
\hline 2MASS J16141107-2305362 & $-2.28(-0.07,+0.12)$ & $30(-8,+9)$ & $1.04(-0.04,+0.04)$ & $3(-1,+1)$ & $4(-3,+48)$ & $46(-40,+104)$ \\
\hline 2MASS J16142029-1906481 & $-1.03(-0.12,+0.17)$ & $29(-2,+1)$ & $1.10(-0.02,+0.06)$ & $9(-5,+1)$ & $27(-23,+10)$ & $19(-19,+32)$ \\
\hline 2MASS J16143367-1900133 & $-2.69(-0.18,+0.29)$ & $11(-9,+13)$ & $1.14(-0.06,+0.05)$ & $8(-6,+5)$ & $69(-43,+18)$ & $51(-38,+109)$ \\
\hline 2MASS J16153456-2242421 & $-1.63(-0.18,+0.11)$ & $21(-2,+2)$ & $1.12(-0.04,+0.06)$ & $3(-2,+16)$ & $46(-21,+12)$ & $170(-31,+10)$ \\
\hline 2MASS J16154416-1921171 & $-0.88(-0.21,+0.25)$ & $10(-1,+2)$ & $1.15(-0.05,+0.05)$ & $9(-1,+8)$ & $40(-17,+24)$ & $117(-54,+26)$ \\
\hline 2MASS J16163345-2521505 & $-2.33(-0.57,+0.53)$ & $72(-23,+25)$ & $1.12(-0.05,+0.06)$ & $1(-1,+2)$ & $88(-9,+2)$ & $64(-9,+9)$ \\
\hline 2MASS J16181904-2028479 & $-1.62(-0.18,+0.29)$ & $11(-3,+6)$ & $1.13(-0.05,+0.06)$ & $8(-5,+5)$ & $56(-46,+7)$ & $90(-56,+42)$ \\
\hline 2MASS J16215466-2043091 & $-3.08(-0.55,+0.48)$ & $8(-8,+29)$ & $1.13(-0.05,+0.05)$ & $1(-1,+8)$ & $82(-53,+8)$ & $127(-110,+41)$ \\
\hline 2MASS J16270942-2148457 & $-1.96(-0.13,+0.25)$ & $22(-6,+10)$ & $1.13(-0.05,+0.05)$ & $8(-6,+7)$ & $70(-33,+15)$ & $176(-29,+25)$ \\
\hline 2MASS J16303390-2428062 & $-2.98(-0.23,+0.27)$ & $96(-66,+3)$ & $1.13(-0.05,+0.05)$ & $1(-1,+8)$ & $74(-25,+16)$ & $76(-47,+75)$ \\
\hline
\end{tabular}

distributed priors. If a disk was not in the Mathews et al. (2013) sample, we assumed a normally distributed prior for $p$, with a mean of 1.13 (the median value of $p$ for their sample of 45 disks) and standard deviation of 0.05 . For $h_{0}$, three-quarters of the Mathews et al. (2013) disks had $h_{0}<10 \mathrm{au}$, so we assumed uniform priors from $0-10 \mathrm{au}$ and 10-20 au, with the probability of $h_{0}>10$ au equal to one-third of the probability of $h_{0}<10$ au

We also assumed a constant dust opacity throughout the disk. Previous multi-wavelength studies of disks at millimeter and centimeter wavelengths suggested radial variations in dust opacity due to grain growth (Isella et al. 2010a; Banzatti 
Table 2

CO Fitting Results

\begin{tabular}{|c|c|c|c|c|c|}
\hline Source & 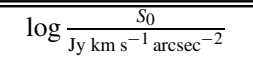 & $\gamma$ & $R_{\mathrm{CO}}(\mathrm{au})$ & Inclination (deg) & Position Angle (deg) \\
\hline 2MASS J15521088-2125372 & $0.77(-0.18,+0.32)$ & $1.71(-1.32,+0.05)$ & $24(-13,+11)$ & $24(-17,+39)$ & $89(-61,+57)$ \\
\hline 2MASS J15530132-2114135 & $0.47(-0.23,+0.51)$ & $1.70(-0.82,+0.08)$ & $17(-17,+37)$ & $88(-61,+2)$ & $70(-64,+47)$ \\
\hline 2MASS J15534211-2049282 & $0.96(-0.21,+0.26)$ & $0.05(-0.05,+0.63)$ & $51(-10,+10)$ & $77(-10,+8)$ & $95(-13,+11)$ \\
\hline 2MASS J15562477-2225552 & $0.26(-0.39,+0.56)$ & $1.67(-0.77,+0.12)$ & $16(-16,+104)$ & $85(-67,+5)$ & $53(-27,+79)$ \\
\hline 2MASS J16001844-2230114 & $1.20(-0.03,+0.04)$ & $1.32(-0.18,+0.11)$ & $68(-14,+16)$ & $24(-10,+27)$ & $90(-39,+41)$ \\
\hline 2MASS J16014086-2258103 & $1.17(-0.31,+0.53)$ & $1.71(-1.19,+0.06)$ & $12(-11,+13)$ & $87(-50,+3)$ & $72(-42,+55)$ \\
\hline 2MASS J16020757-2257467 & $0.77(-0.09,+0.14)$ & $0.94(-0.63,+0.26)$ & $54(-11,+13)$ & $59(-18,+12)$ & $82(-15,+16)$ \\
\hline 2MASS J16035767-2031055 & $0.71(-0.19,+0.33)$ & $1.69(-0.51,+0.07)$ & $37(-7,+182)$ & $55(-38,+23)$ & $22(-95,+30)$ \\
\hline 2MASS J16035793-1942108 & $1.00(-0.08,+0.06)$ & $0.79(-0.58,+0.19)$ & $43(-6,+7)$ & $43(-24,+10)$ & $3(-28,+23)$ \\
\hline 2MASS J16062277-2011243 & $0.35(-0.27,+0.78)$ & $1.70(-1.33,+0.07)$ & $6(-6,+37)$ & $88(-58,+2)$ & $160(-131,+12)$ \\
\hline 2MASS J16075796-2040087 & $1.67(-0.04,+0.04)$ & $0.74(-0.50,+0.31)$ & $34(-2,+6)$ & $52(-5,+4)$ & $1(-5,+5)$ \\
\hline 2MASS J16081566-2222199 & $0.43(-0.12,+0.18)$ & $1.67(-0.34,+0.09)$ & $30(-14,+129)$ & $3(-2,+52)$ & $15(-88,+50)$ \\
\hline 2MASS J16082324-1930009 & $0.87(-0.22,+0.34)$ & $0.95(-0.38,+0.37)$ & $156(-32,+29)$ & $72(-11,+12)$ & $101(-12,+14)$ \\
\hline 2MASS J16090075-1908526 & $0.97(-0.23,+0.17)$ & $1.30(-0.91,+0.26)$ & $72(-22,+42)$ & $50(-41,+10)$ & $95(-40,+62)$ \\
\hline 2MASS J16095933-1800090 & $0.65(-0.13,+0.23)$ & $1.69(-0.96,+0.08)$ & $52(-23,+33)$ & $63(-42,+16)$ & $119(-42,+40)$ \\
\hline 2MASS J16104636-1840598 & $0.45(-0.16,+0.36)$ & $1.70(-0.40,+0.08)$ & $22(-22,+148)$ & $54(-33,+25)$ & $50(-43,+68)$ \\
\hline 2MASS J16115091-2012098 & $0.54(-0.14,+0.56)$ & $1.30(-0.26,+0.26)$ & $75(-29,+241)$ & $86(-36,+4)$ & $26(-13,+13)$ \\
\hline 2MASS J16123916-1859284 & $0.70(-0.07,+0.07)$ & $0.85(-0.13,+0.09)$ & $169(-26,+24)$ & $53(-8,+6)$ & $104(-11,+14)$ \\
\hline 2MASS J16142029-1906481 & $1.52(-0.05,+0.07)$ & $0.97(-0.12,+0.09)$ & $88(-6,+6)$ & $58(-4,+4)$ & $5(-4,+4)$ \\
\hline 2MASS J16143367-1900133 & $1.32(-0.36,+0.35)$ & $1.71(-0.96,+0.07)$ & $14(-12,+12)$ & $83(-51,+5)$ & $88(-50,+76)$ \\
\hline 2MASS J16154416-1921171 & $1.73(-0.01,+0.01)$ & $1.01(-0.01,+0.01)$ & $430(-10,+10)$ & $61(-1,+1)$ & $28(-1,+1)$ \\
\hline 2MASS J16163345-2521505 & $0.79(-0.23,+0.36)$ & $1.31(-0.90,+0.27)$ & $45(-19,+22)$ & $81(-17,+7)$ & $59(-19,+16)$ \\
\hline 2MASS J16181904-2028479 & $0.48(-0.11,+0.28)$ & $1.69(-0.26,+0.07)$ & $26(-11,+176)$ & $69(-60,+5)$ & $155(-28,+59)$ \\
\hline
\end{tabular}

et al. 2011; Guilloteau et al. 2011; Pérez et al. 2012, 2015; Trotta et al. 2013; Menu et al. 2014; ALMA Partnership et al. 2015; Guidi et al. 2016; Tazzari et al. 2016). Similar radial variations may be present in our Upper Sco disks, and, in fact, are predicted by models of dust transport and evolution (e.g., Dullemond \& Dominik 2005; Birnstiel et al. 2010). However, there is no way to constrain the dust opacity based on our single-wavelength data. Thus, we used a uniform dust opacity, calculated as a function of wavelength using Mie theory for dust grains composed of a mix of carbons, ices, and silicates (e.g., Pollack et al. 1994), with a grain size distribution of $n(a) \propto a^{-3.5}$ and a maximum grain size of $1 \mathrm{~cm}$. Only $\Sigma_{0}$, which is inversely proportional to opacity for a given flux density, was sensitive to our choice of maximum grain size.

The Monte Carlo radiative transfer code RADMC-3D (Dullemond 2012) was used to determine the temperature throughout the model dust disk due to stellar irradiation. We adopted the stellar parameters derived in Paper I. We assumed a minimum temperature of $10 \mathrm{~K}$ at any location in the disk to account for other heat sources such as radioactive decay and cosmic rays (e.g., D'Alessio et al. 2001; Woitke 2015). RADMC-3D was then used to generate an image of the model disk for a given inclination and position angle. The Fourier transform of this image provided a grid of model visibilities, which was interpolated at our observational $u v$ points. We used the $\chi^{2}$ difference between the model and observed visibilities (real and imaginary) to calculate the likelihood of the current set of model parameters:

$$
L=\sum \exp \left[-\frac{\left(\operatorname{Re}_{\mathrm{mod}}-\mathrm{Re}_{\mathrm{obs}}\right)^{2}}{2 \sigma_{\mathrm{vis}}^{2}}-\frac{\left(\mathrm{Im}_{\mathrm{mod}}-\mathrm{Im}_{\mathrm{obs}}\right)^{2}}{2 \sigma_{\mathrm{vis}}^{2}}\right],
$$

where $\frac{1}{\sigma_{\text {vis }}^{2}}$ is the visibility weight. The observed visibilities were corrected to center the disk at the phase center of observations using the disk positions determined in Paper I. The Markov chain Monte Carlo implementation emcee (Foreman-Mackey et al. 2013) was used to constrain $\Sigma_{0}, R_{\text {dust }}, h_{0}, p$, inclination, and position angle.

Table 1 contains the most likely values for $\Sigma_{0}, R_{\text {dust }}, h_{0}, p$, inclination, and position angle from the continuum fitting, together with their uncertainties. The values of each parameter sampled by the MCMC in the fitting of each source gave the final probability distribution of that parameter. The reported values in Table 1 were taken from the peak of these distributions. The uncertainties were defined as the bounds of the range around the peak containing $68.3 \%$ of the integrated probability. The dust disks range from 4 to 173 au in radius, although $82 \%$ of the disks have radii less than 50 au. Figure 1 shows an image of the bestfit model of each source, along with the observed image and residuals. This figure also shows the deprojected observed and best-fit model visibilities as a function of baseline length.

Five sources, 2MASS J16032225-2413111, 2MASS J16054540-2023088, 2MASS J16111330-2019029, 2MASS J16123916-1859284, and 2MASS J16135434-2320342, exhibited $5 \sigma$ emission in the residual images of their best-fit models. We fit point-source models to the residual visibilities of these sources; their continuum flux densities and positions relative to the primary disks are listed in Table 3. The NASA/IPAC Extragalactic Database lists no known background galaxies at the positions of these secondary sources. 2MASS J160545402023088 was identified as a double line spectroscopic binary by Dahm et al. (2012), while none of the other sources with secondary emission are known binaries.

The secondary sources of 2MASS J16032225-2413111, 2MASS J16123916-1859284, and 2MASS J16135434-2320342 were also identified in Paper I. The secondary sources of 2MASS J16054540-2023088 and 2MASS J16111330-2019029 were too close to their respective primary sources to have been identified 
Observations
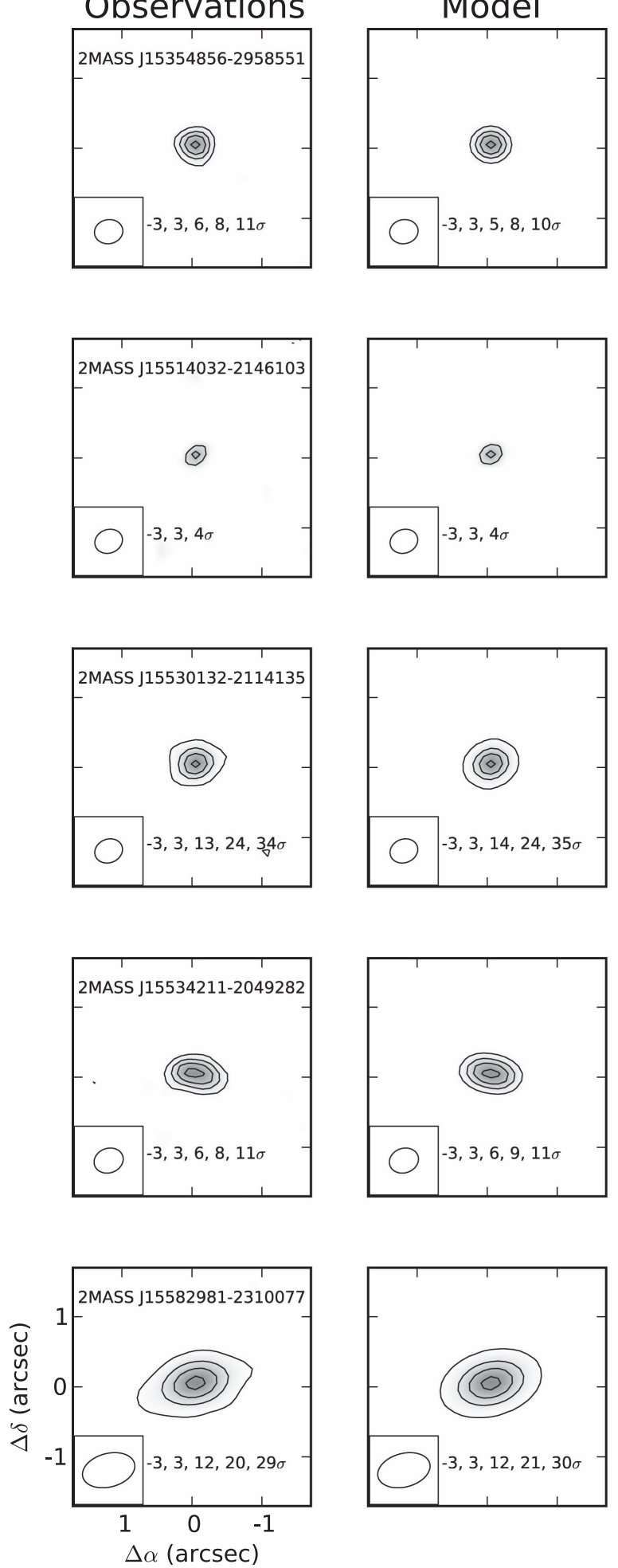

(a)
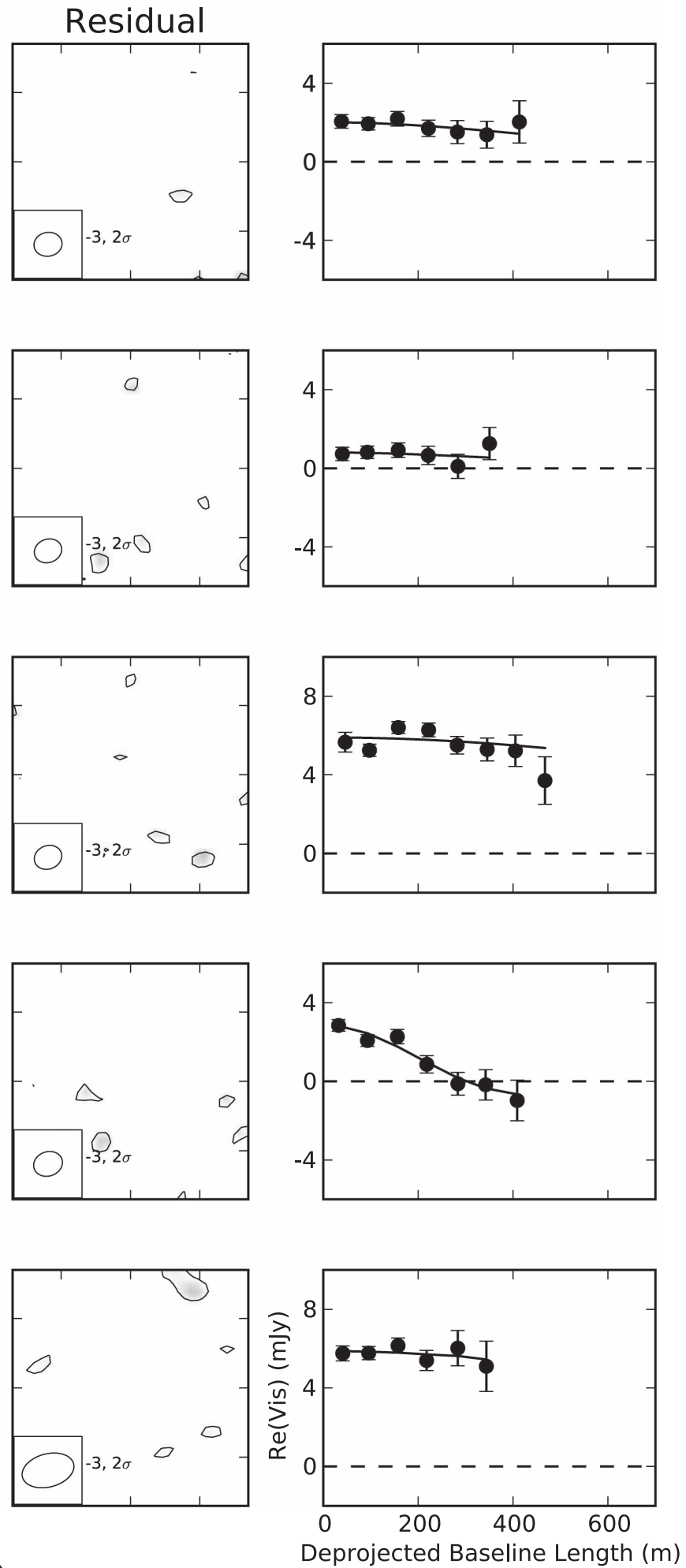

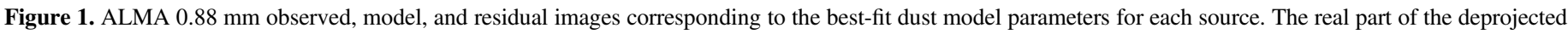

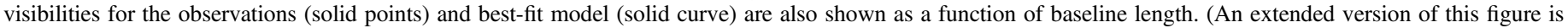
available.)

in Paper I, but can be seen in Figure 1 as non-axisymmetric extended emission on the eastern side of the primaries. The secondary source to 2MASS J16135434-2320342, by far the brightest in our sample, is clearly visible as a second disk to the east of the primary. We used the MCMC fitting method described above to determine the dust properties of this source after subtracting our best-fit model for the primary disk from he observed visibilities and shifting the phase center to the secondary disk. The best-fit parameters are given in Table 1 for both components of 2MASS J16135434-2320342. 
Table 3

Secondary Source Properties

\begin{tabular}{|c|c|c|c|}
\hline Field & $S_{\text {tot }}(\mathrm{mJy})$ & $\Delta \alpha(\operatorname{arcsec})$ & $\Delta \delta(\operatorname{arcsec})$ \\
\hline 2MASS J16032225-2413111 & $0.85 \pm 0.14$ & $0.80 \pm 0.04$ & $0.06 \pm 0.04$ \\
\hline 2MASS J16054540-2023088 & $1.00 \pm 0.15$ & $0.39 \pm 0.04$ & $-0.01 \pm 0.04$ \\
\hline 2MASS J16111330-2019029 & $1.00 \pm 0.16$ & $0.48 \pm 0.04$ & $-0.19 \pm 0.04$ \\
\hline 2MASS J16123916-1859284 & $1.09 \pm 0.16$ & $0.75 \pm 0.04$ & $-0.15 \pm 0.04$ \\
\hline 2MASS J16135434-2320342 & $5.83 \pm 0.12$ & $0.59 \pm 0.03$ & $-0.18 \pm 0.03$ \\
\hline
\end{tabular}

\section{CO Modeling}

\subsection{CO Surface Brightness Fitting}

Our modeling approach for the $\mathrm{CO}$ data was similar to that for the continuum. We used the continuum-subtracted visibilities for each of the $23 \mathrm{CO}$ detections to measure the radial extent of gas. Due to the likelihood of optically thick $\mathrm{CO}$ emission, we fit the $\mathrm{CO}$ surface brightness of the disks instead of a physical surface density. For each source, we used the velocity range corresponding to the $J=3-2$ emission line, as determined by Paper I, to generate integrated "moment 0 visibilities." We then fit to the real and imaginary part of these visibilities as described in Section 3. For consistency with our continuum fitting, we assumed an azimuthally symmetric disk with surface brightness described by a truncated power law,

$$
S(R)=S_{0}\left(\frac{R}{10 \mathrm{au}}\right)^{-\gamma}
$$

for $R<=R_{\mathrm{CO}}$ and $S=0$ beyond $R_{\mathrm{CO}}$. We used emcee to fit for the surface brightness normalization, $S_{0}$, the power-law slope, $\gamma$, the outer radius, $R_{\mathrm{CO}}$, the inclination, and position angle.

Table 2 presents the best-fit parameters of our $\mathrm{CO}$ model fitting. Best-fit values and uncertainties were defined as described in Section 3 for the dust modeling. Observed, model, and residual images for the $\mathrm{CO}$ are shown in Figure 2, along with the deprojected observed and best-fit visibilities of each source. We found CO outer radii ranging from 6 to $430 \mathrm{au}$. 2MASS J16154416-1921171 was the largest CO disk in our sample, with a radius of $430 \mathrm{au}$. Examination of the CO channel maps suggested contamination by a surrounding molecular cloud and we therefore excluded this source from further $\mathrm{CO}$ analysis. With this source excluded, our largest $\mathrm{CO}$ outer radius is $169 \mathrm{au}$. It is worth noting that the gaseous disks may extend beyond our measured $\mathrm{CO}$ outer radii; $\mathrm{CO}$ will be subject to freeze-out and photodissociation in the outer parts of disks (though some $\mathrm{CO}$ will return to the gas phase through non-thermal desorption, e.g., Öberg 2016), while $\mathrm{H}_{2}$ and other gaseous molecules can survive out to these regions.

\subsection{Expected CO Fluxes}

Only 21 of the 55 continuum-detected sources in our sample were also detected in $\mathrm{CO}$. The relatively low number of $\mathrm{CO}$ detections suggested that the $\mathrm{CO}$ is either heavily depleted relative to the dust or has a compact emitting area due to small disk sizes. In this section, we test the latter possibility. We used the results of our continuum modeling to predict the $\mathrm{CO}$ $J=3-2$ line flux from our continuum-detected disks assuming the gas and dust share the same spatial distribution.

To estimate the expected $\mathrm{CO} J=3-2$ line fluxes for our continuum-detected disks, we used the posterior distributions of $\Sigma_{0}, R_{\text {dust }}, h_{0}, p$, inclination, and position angle from our
MCMC continuum fits to generate a sample of model dust disks for each source. We then added $\mathrm{CO}$ to these disks by assuming that the $\mathrm{CO}$ and dust share the same temperature structure and spatial distribution with a gas-to-dust ratio of 100 and a $\mathrm{CO}$ to $\mathrm{H}_{2}$ ratio of $7 \times 10^{-5}$ by number (Beckwith \& Sargent 1993; Dutrey et al. 1996, and references therein). If a source was detected in $\mathrm{CO}$, we sampled $\mathrm{CO}$ outer radii $\left(R_{\mathrm{CO}}\right)$ from the posterior distribution of our surface brightness fitting and extended the model $\mathrm{CO}$ disk out to these radii. If $R_{\mathrm{CO}}$ is larger than $R_{\text {dust }}$, we used our $3 \sigma$ upper limits on the total continuum flux between $R_{\text {dust }}$ and $R_{\mathrm{CO}}$ to calculate an upper limit on the dust mass in this annulus, assuming optically thin emission and a dust temperature of $10 \mathrm{~K}$. This dust mass upper limit was then converted into a uniform dust surface density between $R_{\text {dust }}$ and $R_{\mathrm{CO}}$, and the disk was populated with $\mathrm{CO}$ as described above.

We took into account the removal of $\mathrm{CO}$ from the gas phase by freeze-out and photodissociation. At any location in a model disk where the temperature was less than $20 \mathrm{~K}$, we assumed the $\mathrm{CO}$ was frozen onto dust grains and had an abundance of zero (Collings et al. 2003; Bisschop et al. 2006). While a small fraction of this $\mathrm{CO}$ will re-enter the gas phase through UV photodesorption (Öberg et al. 2009a, 2009b; Fayolle et al. 2011; Chen et al. 2014) and cosmic-ray heating (Hasegawa \& Herbst 1993), modeling of these processes has shown the effects on CO observations to be negligible (Öberg et al. 2015). A common method for treating photodissociation in disks is to assume a minimum column density of $\mathrm{H}_{2}$ that will shield CO from destruction by stellar and interstellar ultraviolet and X-ray radiation. Visser et al. (2009) modeled a molecular cloud exposed to the interstellar radiation field and found that an $\mathrm{H}_{2}$ column density of $10^{21} \mathrm{~g} \mathrm{~cm}^{-2}$ would shield $\mathrm{CO}$. Detailed modeling (Aikawa \& Nomura 2006; Gorti \& Hollenbach 2008) and observations (Qi et al. 2011) of photodissociation in disks around accreting young stars found similar results. Thus, it is often assumed that $\mathrm{CO}$ in disks will only survive below a vertical column density of $10^{21} \mathrm{~g} \mathrm{~cm}^{-2}$ of $\mathrm{H}_{2}$ (Williams \& Best 2014; Walsh et al. 2016).

In the young circumstellar disks modeled in this way, high energy radiation produced by stellar accretion dominated over the interstellar radiation field (van Zadelhoff et al. 2003; Visser et al. 2009), providing an abundant source of UV and X-ray photons. This may not be the case for the more evolved disks in Upper Sco, however. Dahm \& Carpenter (2009) found that only 7 out of a sample of 35 disk-bearing Upper Sco sources showed signs of accretion, and that the median accretion rate of these 7 sources was an order of magnitude lower than that of younger disks in Taurus. Therefore, the disks in the present sample are likely to be exposed to much weaker radiation fields than younger, more strongly accreting disks, and will require less material to shield CO. To reflect this uncertainty in the minimum shielding column density required, we treated photodissociation in two ways. First, we followed the typical 
Observations
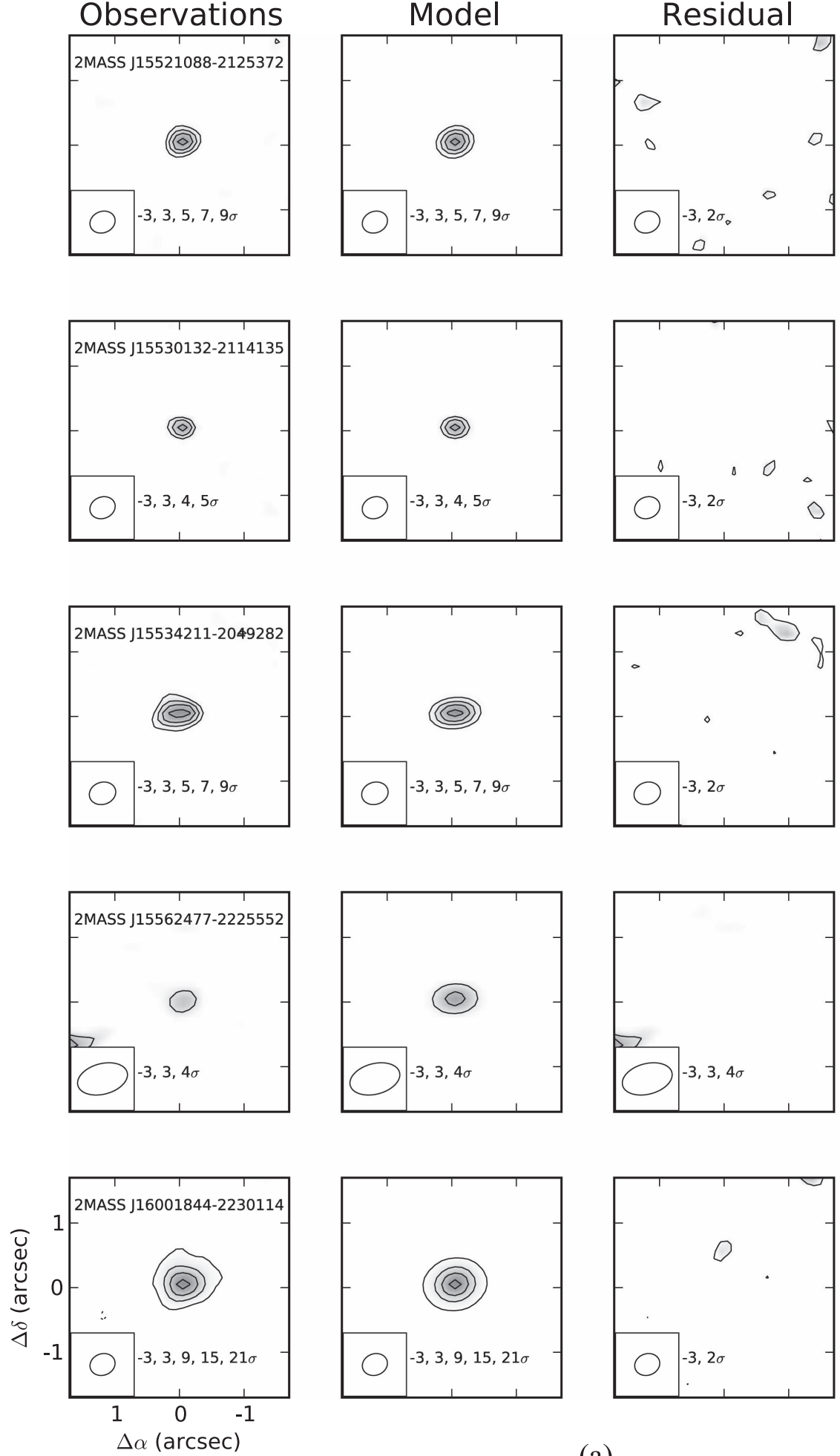

(a)
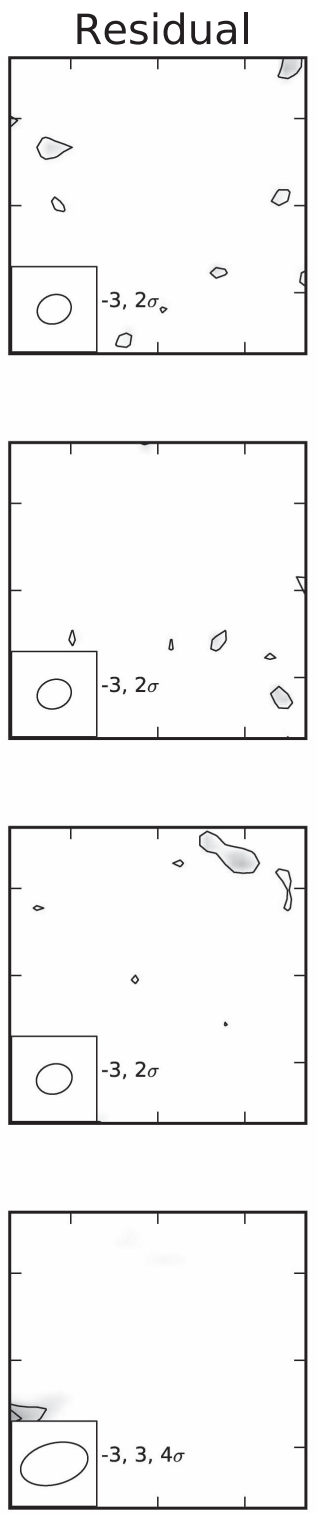
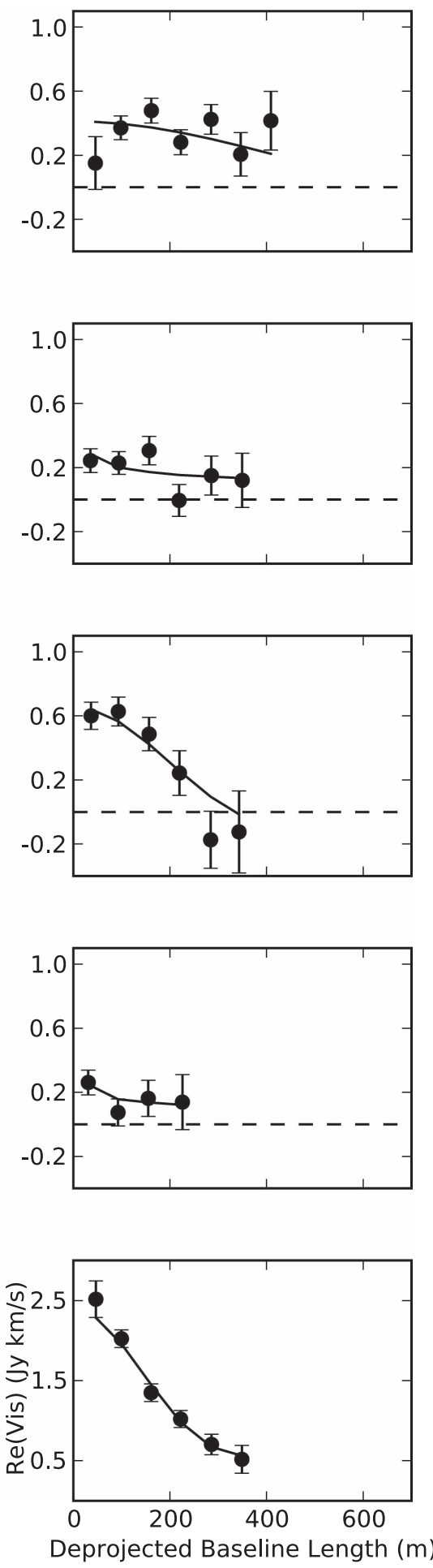

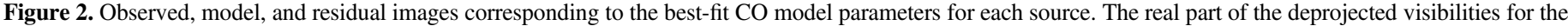
observations (solid points) and best-fit model (solid curve) are also shown as a function of baseline length. (An extended version of this figure is available.)

assumption for younger disks and assumed that if the vertical column density of $\mathrm{H}_{2}$ above any location in the disk was below $10^{21} \mathrm{~cm}^{-2}$, CO would be photodissociated to a density of zero. As an alternative, we also calculated the expected $\mathrm{CO}$ flux without any photodissociation, which we consider a conservative upper limit on the amount of gaseous $\mathrm{CO}$ that survives and therefore on the model CO flux. We note that once enough CO survives to become optically thick, the shielding column density and precise amount that survives has little impact on the expected flux. Ignoring photodissociation entirely is an approximation of this scenario.

With these model CO disks, we used RADMC3D to calculate the $\mathrm{CO} J=3-2$ flux over the velocity range determined for each source in Paper I. We repeated this 

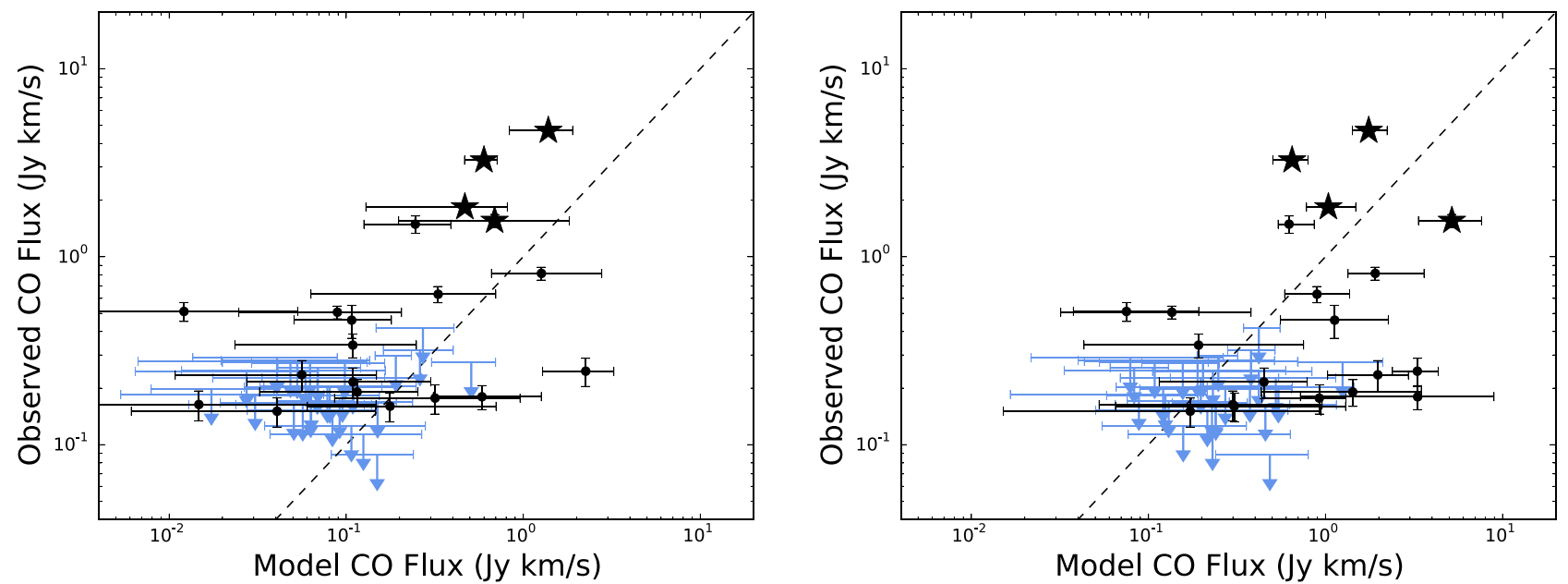

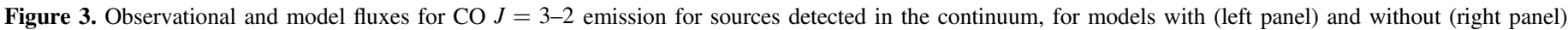

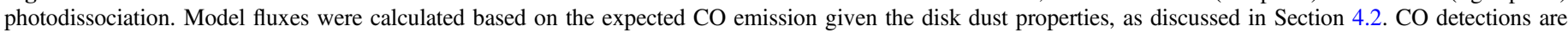

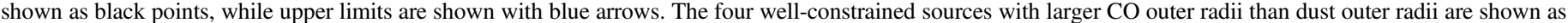

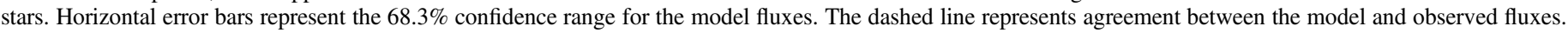

process for every continuum-detected source in our sample, generating a distribution of model $\mathrm{CO}$ fluxes for each. Figure 3 shows a comparison of our model CO fluxes with our observed fluxes from Paper I for the two treatments of photodissociation described above. Among the sources detected in $\mathrm{CO}$, there was considerable scatter in the observed fluxes relative to the model fluxes. This reflects the uncertainties of our modeling procedure, both statistical from our uncertain dust model parameters and systematic relating to our assumptions regarding gas temperature and gas-to-dust ratio, as well as our treatments of freeze-out and photodissociation. Without photodissociation, $\mathrm{CO}$ fluxes increased by as much as an order of magnitude. This was due to cases where the combination of freeze-out and photodissociation truncated the $\mathrm{CO}$ disk inside of the observed $R_{\mathrm{CO}}$, reducing the emitting area of the disk. For the sources not detected in $\mathrm{CO}$, the model fluxes were consistent with observational upper limits.

\section{Discussion}

\subsection{Dust Disk Sizes}

Based on our derived dust outer radii, the majority of the continuum-detected dust disks in our sample are quite compact. Empirically, we determined that to constrain the dust outer radius $\left(R_{\text {dust }}\right)$ to better than a factor of 2 required a signal-tonoise of at least 15 . The 25 disks that meet this threshold have dust outer radii ranging from 8 to $65 \mathrm{au}$, with a median of 21 au. Only two disks, 2MASS J16082324-1930009 and 2MASS J16090007-1908526, have radii larger than $50 \mathrm{au}$. Note that this excludes 2MASS J15583692-2257153, 2MASS J16042165-2130284, and 2MASS J16113134-1838259 (see Section 2), all of which appear to be larger than 65 au in radius based on visual inspection of their continuum images (see Paper I). Figure 4 shows the posterior probability distributions of the outer radius for the 25 high signal-to-noise disks. The distributions are sharply peaked around the best-fit value, with no significant probability tails extending out to larger radii. Thus, while we cannot rule out that the dust surface density follows a different distribution than $R^{-1}$, such as a power law with a different slope or with an exponential tail, any such distribution must fall off rapidly at or near our best-fit outer radii.

While we lack a sample of younger disks analyzed in the same way to compare with our 5-11 Myr old Upper Sco sample, we do see evidence that the dust disks in Upper Sco are more compact than younger disks. Tripathi et al. (2017) measured the sizes of 50 disks primarily located in the 1-2 Myr old Taurus and Ophiuchus star-forming regions by fitting "Nuker" profiles (Lauer et al. 1995) to the continuum emission. More than half of the stars in this sample have spectral types earlier than K9, compared to only 2 of 25 stars in our high signal-to-noise sample, J16141107-2305362 and 2MASS J16154416-1921171. We therefore include only spectral types K9-M5 when comparing the present Upper Sco sample to these younger disks. Pre-main-sequence stars of these spectral types are fully convective and evolve at approximately constant temperature (e.g., Siess et al. 2000), making spectral type a good proxy for stellar mass even when comparing stars of different ages. For this spectral type range, Tripathi et al. (2017) found effective radii, defined as the radius containing $68 \%$ of the disk continuum flux, ranging from 19 to $182 \mathrm{au}$, with a median of $48 \mathrm{au}$. Assuming optically thin dust emission and constant midplane dust temperature in the outer regions of our Upper Sco disks, where most of the dust mass resides, we can define an effective radius for our sample as containing $68 \%$ of the total dust mass, which will be approximately equivalent to the radius containing $68 \%$ of the continuum flux. With this definition, the effective radii of our high signal-to-noise sources with spectral types K9-M5 range from 5 au to 44 au, with a median of $14 \mathrm{au}$. These effective radii may in fact be overestimated for the Upper Sco disks, as the innermost region of the disks will have higher dust temperatures than the outer regions, causing the disk continuum flux to be slightly more concentrated at small radii than the dust mass. However, since we found that the disks in Upper Sco appear to be smaller than the younger disks of Tripathi et al. (2017), this effect strengthens our conclusions.

In a separate study of young disks, Tazzari et al. (2017) fit for the outer radii of 22 disks in the 1-3 Myr old Lupus starforming region. The authors used a power law with an exponential cutoff to parameterize the dust surface density, 

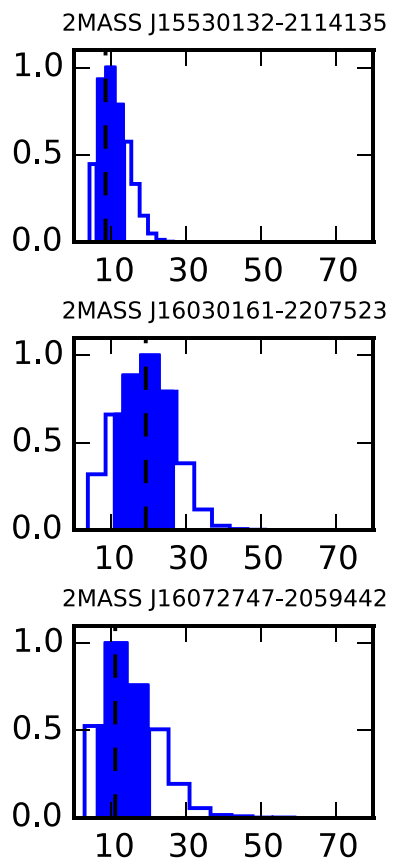

2MASS J16135434-2320342B

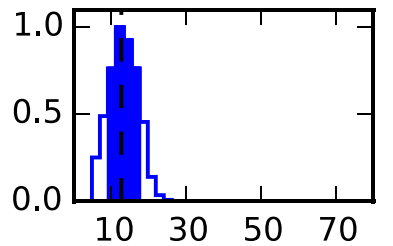

2MASS J16141107-2305362

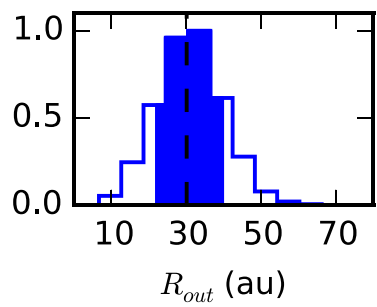

2MASS J16001844-2230114

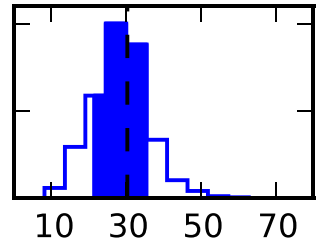

2MASS J16032225-2413111
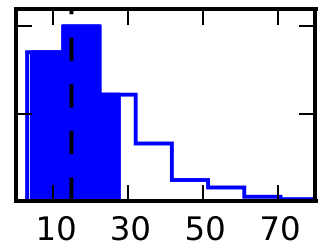

2MASS J16075796-2040087
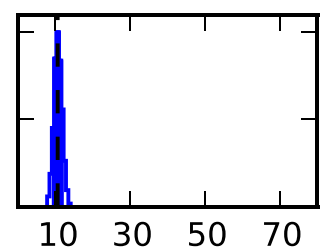

2MASS J16153456-2242421

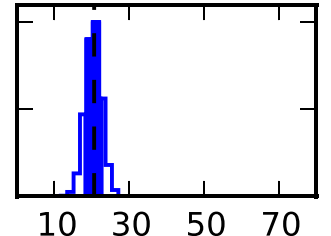

2MASS J15582981-2310077

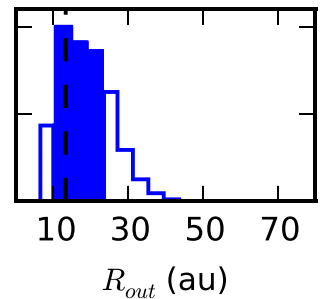

2MASS J16014086-2258103

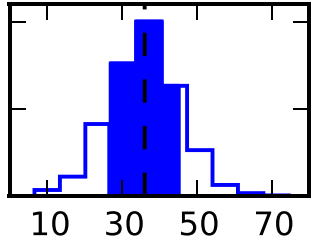

2MASS J16054540-2023088

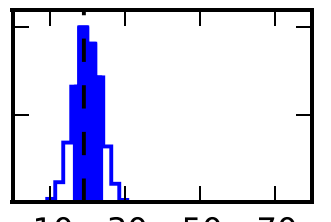

$\begin{array}{llll}10 & 30 & 50 & 70\end{array}$

2MASS J16111330-2019029

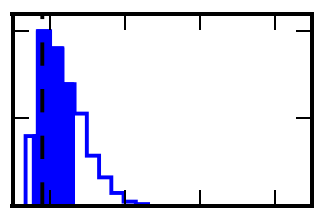

$\begin{array}{llll}10 & 30 & 50 & 70\end{array}$

2MASS J16154416-1921171

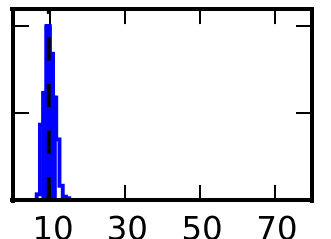

MASS J16082324-1930009

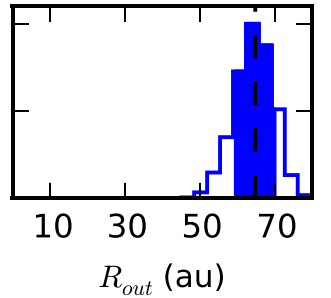

2MASS J16020757-2257467

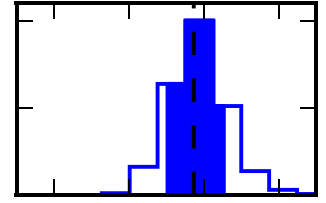

$\begin{array}{llll}10 & 30 & 50 & 70\end{array}$

2MASS J16064102-2455489

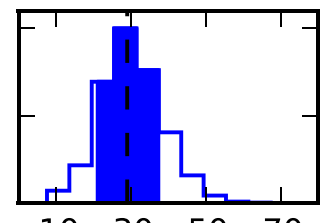

$\begin{array}{llll}10 & 30 & 50 & 70\end{array}$

2MASS J16123916-1859284

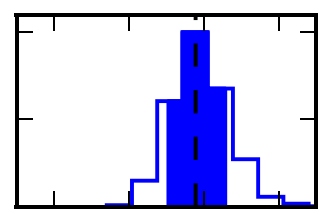

$\begin{array}{llll}10 & 30 & 50 & 70\end{array}$

2MASS J16181904-2028479

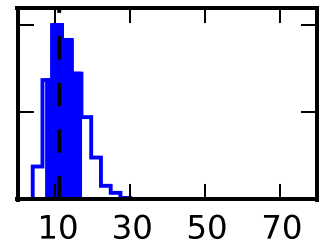

MASS J16090075-1908526

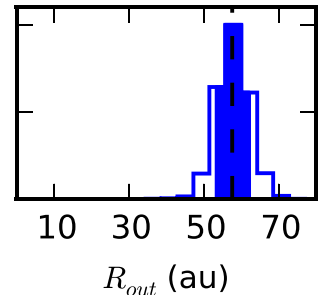

2MASS J16024152-2138245

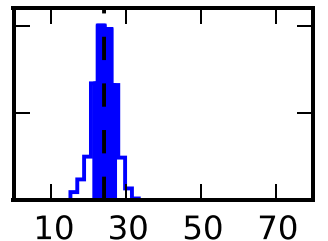

2MASS J16072625-2432079

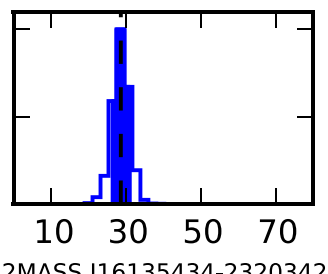

MASS J16135434-2320342
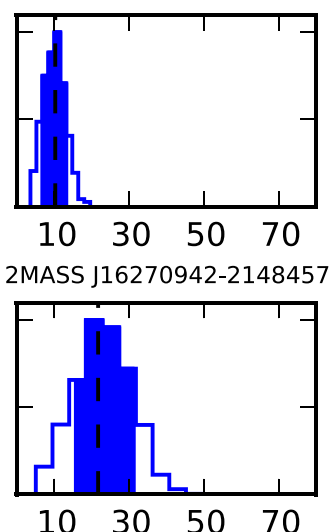

2MASS J16142029-1906481

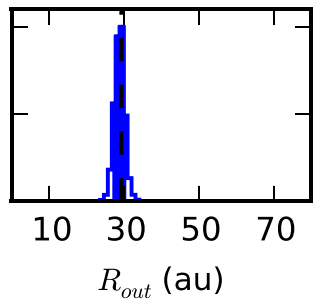

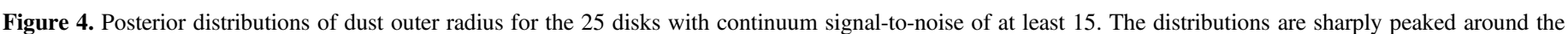

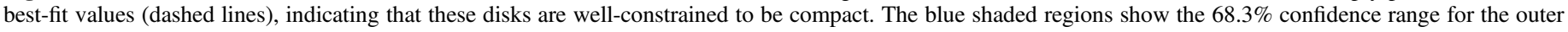
radii.

defining the effective radius as that which contains $95 \%$ of the dust mass. We again exclude 2MASS J16141107-2305362 and 2MASS J16154416-1921171, restricting our comparison to stars between 0.15 and $0.7 M_{\odot}$. For this stellar mass range, Tazzari et al. (2017) measure effective radii ranging from 18 to $129 \mathrm{au}$, with a median of $55 \mathrm{au}$. Calculating the radii of our disks containing $95 \%$ of the dust mass, we find a range of 7-62 au, with a median of 20 au. Taken at face-value, these results suggest that the disks in Upper Sco are smaller than those found in Taurus, Ophiuchus, and Lupus by a factor of $\sim 3$. However, we caution that a self-consistent analysis of all these disks needs to be performed to confirm this trend.

Finally, we note that Hendler et al. (2017) measured dust outer radii from the spectral energy distributions of 11 young disks around very low mass stars and brown dwarfs in the Taurus and Chamaeleon I star-forming regions, finding disk sizes similar to those we see in Upper Sco. This younger sample probes lower stellar masses than the present Upper Sco sample, and is therefore not directly comparable. However, van der Plas et al. (2016) used ALMA to image the disks around seven very low mass stars and brown dwarfs in Upper Sco. None of these objects were spatially resolved, constraining them to also be compact ( $\lesssim 40 \mathrm{au})$. Follow-up studies of these low mass stellar and substellar systems can be used to determine if the reduction in dust disk sizes with age observed here extends to lower stellar masses.

\subsection{Comparing Dust and CO}

Empirically, we found that to measure $R_{\mathrm{CO}}$ to better than a factor of 2 required a $\mathrm{CO}$ signal-to-noise of at least 8 , as measured from the moment 0 maps in Paper I. This threshold is lower than that of continuum data due to the $\mathrm{CO}$ model fitting having one less free parameter than the continuum fitting. Also, the $\mathrm{CO}$ emission tends to be more extended than the continuum emission, allowing for a smaller fractional uncertainty on the outer radius for a given signal-to-noise. For the nine disks with well-constrained $\mathrm{CO}$ outer radii we measured radii ranging from 12 to $169 \mathrm{au}$, with a median of 54 au, excluding 2MASS 

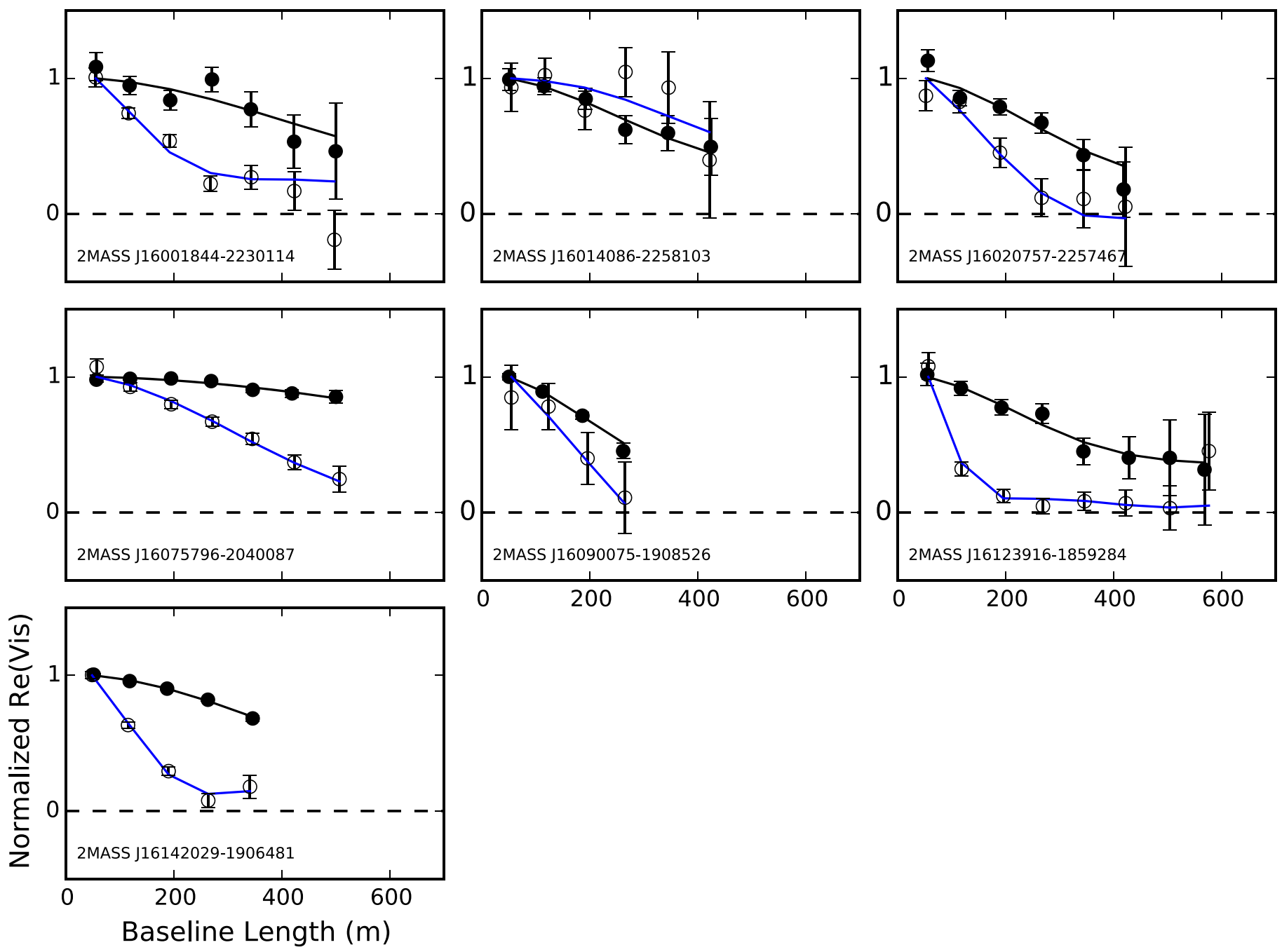

Figure 5. Continuum (black circles) and $\mathrm{CO}$ (open circles) deprojected visibilities for the sources with well-constrained dust and CO outer radii. The black and blue curves show the best-fit models for the dust and CO, respectively. Four sources, 2MASS J16001844-2230114, 2MASS J16075796-2040087, 2MASS J161239161859284, and 2MASS J16142029-1906481, exhibited detectable CO emission extending beyond their dust emission.

J16154416-1921171 (see Section 4.1). Only three of these nine sources have $\mathrm{CO}$ radii less than 50 au. Figure 5 displays the continuum and $\mathrm{CO}$ deprojected visibilities, with their best-fit models, for the seven sources with well-constrained dust and $\mathrm{CO}$ outer radii. Figure 6 shows the outer radii for these sources. Four sources, 2MASS J16001844-2230114, 2MASS J160757962040087, 2MASS J16123916-1859284, and 2MASS J161420291906481, have detectable CO emission extending to larger radii than the detectable dust emission. Previous observations of younger disks also revealed $\mathrm{CO}$ emission extending beyond any detectable continuum emission (e.g., Piétu et al. 2005; Isella et al. 2007; Panić et al. 2009; Andrews et al. 2012) and enhanced gas-todust ratios at large radii (Isella et al. 2016).

However, optical depth effects must be taken into account (Hughes et al. 2008; Facchini et al. 2017). A low surface density tail of dust may extend beyond the apparent dust outer radius, with its optically thin emission undetected. Emission from $\mathrm{CO}$, on the other hand, is optically thick down to low surface densities, and therefore is more likely to be detected in the outer parts of a disk even if the dust emission is weak. To test this possibility for the four sources with $\mathrm{CO}$ potentially extending beyond the dust, we used the predicted CO fluxes of Section 4.2 and Figure 3, where these four sources are shown as stars. These models assumed a low surface density tail of dust, consistent with our upper limits, between the apparent dust and $\mathrm{CO}$ outer radii, with standard $\mathrm{CO}$ abundances relative to the dust. We found that the predicted CO line fluxes of these sources were consistent with the observed fluxes, although this was dependent on the assumed photodissociation prescription. Therefore, while these disks may in fact have an enhanced gas-to-dust ratio in the outer disk due to inward grain migration (e.g., Birnstiel \& Andrews 2014), we could not rule out a standard gas-to-dust ratio, with a drop in surface density of both gas and dust beyond the apparent dust outer radius. Further observations that place deeper limits on the dust surface density in the outer disk and/or include the optically thin isotopologues of CO to estimate gas surface densities can be used to distinguish between these two cases.

Previous studies of 1-3 Myr old disks have found evidence for a low $\mathrm{CO}$ abundance relative to dust throughout the disk (Dutrey et al. 2003; Chapillon et al. 2008; Williams \& Best 2014; Hardy et al. 2015; Long et al. 2017). Ansdell et al. (2016) used observations of optically thin ${ }^{13} \mathrm{CO}$ and $\mathrm{C}^{18} \mathrm{O}$ emission to measure the gas-to-dust ratios of 62 disks in 1-3 Myr old Lupus complex, finding that the majority of 


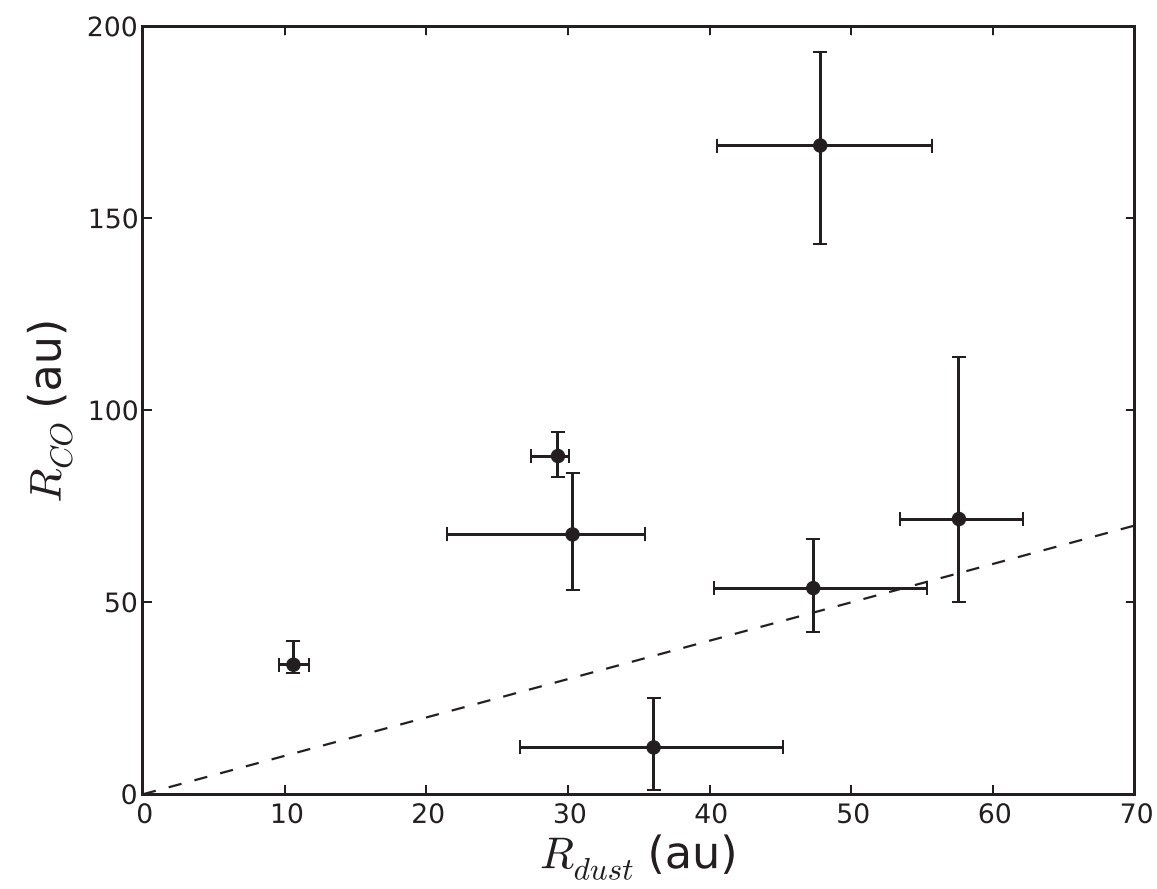

Figure 6. Best-fit CO and dust outer radii for sources where both are well constrained. Four sources, 2MASS J16001844-2230114, 2MASS J16075796-2040087, 2MASS J16123916-1859284, and 2MASS J16142029-1906481, have CO outer radii larger than their dust outer radii.

sources had a gas-to-dust ratio below the ISM value of 100 assuming an ISM abundance of $\mathrm{CO}$ relative to $\mathrm{H}_{2}$ (see also Miotello et al. 2017). Ansdell et al. (2017) found similar results for the 3-5 Myr old $\sigma$ Orionis region. To the extent that $\mathrm{CO}$ traces the total gas mass, this has important implications for disk evolution and the relative timescales of gas and dust dissipation in disks. However, chemical processing of the gas in disks is expected to lower the $\mathrm{CO}$ to $\mathrm{H}_{2}$ ratio (Kama et al. 2016; Yu et al. 2017). Gas mass measurements using the HD $112 \mu \mathrm{m}$ line showed that CO in disks may be depleted relative to hydrogen by up to two orders of magnitude (Bergin et al. 2013; McClure et al. 2016).

If the processes causing these low $\mathrm{CO}$ abundances continue to the age of Upper Sco, this could explain the lack of $\mathrm{CO}$ detections in over half of our continuum-detected disks. However, as our analysis in Section 4.2 shows, CO depletion is not required to explain these non-detections, given the signal-to-noise of the data. The small sizes of these disks alone are sufficient to explain the lack of detectable $\mathrm{CO}$ emission. For the sources where we do see $\mathrm{CO}$ emission, we could not constrain the total mass in $\mathrm{CO}$ due to the likelihood that the emission is optically thick. Upper Sco represents a crucial data point to study the relative evolution of gas and dust in disks, but to do so requires additional sensitivity and/or observations of ${ }^{13} \mathrm{CO}$ and $\mathrm{C}^{18} \mathrm{O}$ to constrain the gaseous $\mathrm{CO}$ mass.

\subsection{Implications for Disk Evolution}

The evolved nature of the disks in our Upper Sco sample presents an opportunity to use the properties of these disks to improve our understanding of disk evolution. Paper I and Ansdell et al. (2016) showed that the dust disks in Upper Sco are on average a factor of 3-4.5 less massive than those in Taurus and Lupus (see also Pascucci et al. 2016). Taken at face-value, the indication of dust disks being more compact as well in Upper Sco (Section 5.1) implies that at least some of this mass is lost through the disappearance of millimeter grains in the outer disk. These grains may be completely removed from the millimeter grain population of the system, either through photoevaporation of the outer disk (e.g., Owen et al. 2012; Alexander et al. 2014; Gorti et al. 2015) or through growth into larger bodies (Testi et al. 2014, and references therein). On the other hand, inward migration of grains from the outer disk (e.g., Weidenschilling 1977; Birnstiel \& Andrews 2014) may cause the inner disk to become optically thick, in effect hiding the dust mass of the outer disk and making the disk appear to be less massive.

We tested these scenarios by comparing the dust surface densities of the Upper Sco disks in this work and of the Lupus disks measured by Tazzari et al. (2017) to determine if the amount of inner disk dust has increased by the age of Upper Sco. We used the best-fit surface density normalizations of both studies, $\Sigma_{0}$, representing the surface density at the normalization radius of $10 \mathrm{au}$, as a proxy for inner disk surface density. The $\Sigma_{0}$ values of Tazzari et al. (2017) are measured assuming a dust opacity at $890 \mu \mathrm{m}$ of $3.37 \mathrm{~cm}^{2} \mathrm{~g}^{-1}$ so we scaled their surface densities to match our assumed dust opacity of $4.94 \mathrm{~cm}^{2} \mathrm{~g}^{-1}$. In addition, the authors report the inferred gas surface density at 10 au assuming a gas-to-dust ratio of 100 . We therefore divided by their assumed gas-to-dust ratio to recover the dust surface density. Applying these corrections and restricting our comparison to stars between 0.15 and $0.7 M_{\odot}$ as in Section 5.1, we found $\Sigma_{0}$ values ranging from $4.9 \times 10^{-3} \mathrm{~g} \mathrm{~cm}^{-2}$ to $0.71 \mathrm{~g} \mathrm{~cm}^{-2}$ for Lupus and $6.2 \times 10^{-3}$ $\mathrm{g} \mathrm{cm}^{-2}$ to $0.23 \mathrm{~g} \mathrm{~cm}^{-2}$ for the high signal-to-noise Upper Sco disks. The mean of $\log \Sigma_{0}$ is -1.04 for Lupus, with a standard deviation of 0.64 , while Upper Sco has a mean $\log \Sigma_{0}$ of -1.65 , with a standard deviation of 0.42 . In addition, the mean $\log \Sigma_{0}$ value we find for Upper Sco implies an inner disk that only becomes optically thick inside of $\sim 1$ au, assuming $\Sigma \propto R^{-1}$. We therefore see no evidence of inner disks increasing in dust surface density between Lupus and Upper Sco. 


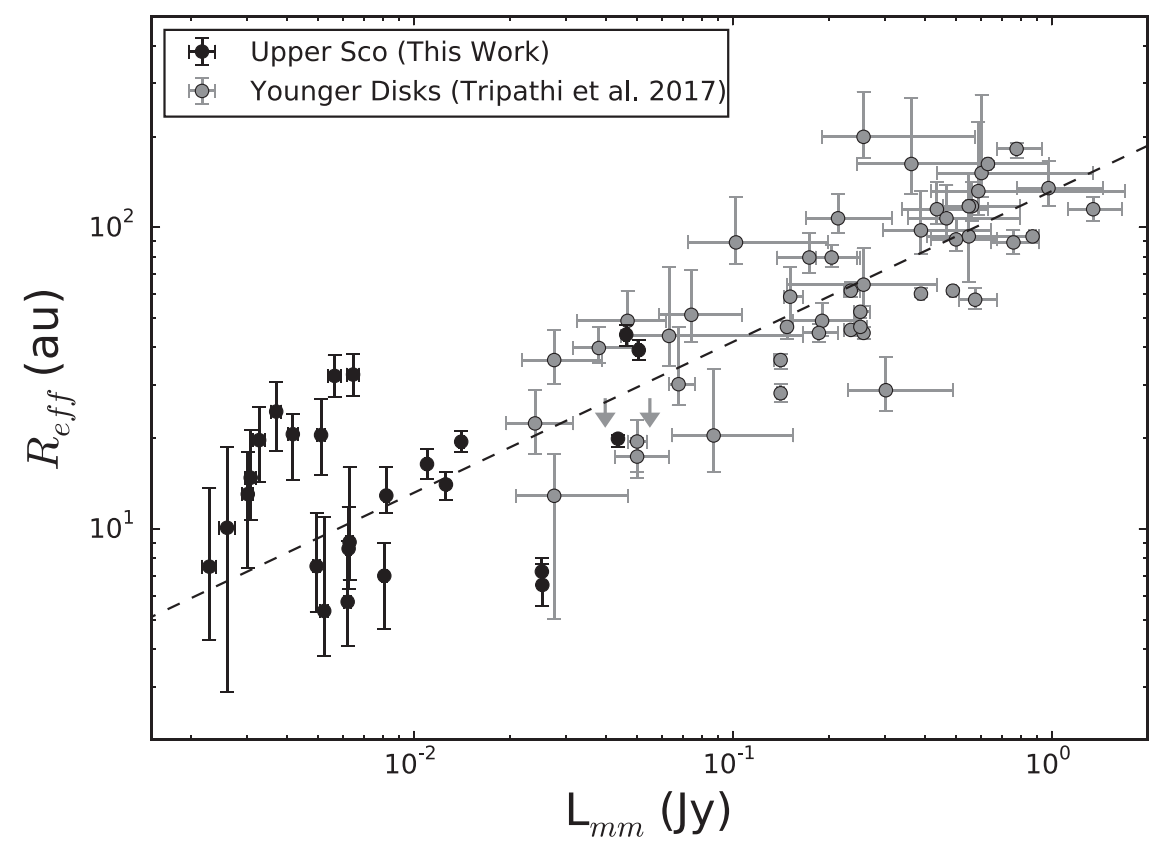

Figure 7. Dust effective radius vs. continuum flux density at $0.88 \mathrm{~mm}$ for circumstellar disks in Taurus and Ophiuchus (gray points, Tripathi et al. 2017) and Upper Sco (black points, this work). Flux densities have been scaled to a distance of $140 \mathrm{pc}$. The dashed line shows the disk size-luminosity relation from Tripathi et al (2017), which has been extrapolated to the flux densities of the Upper Sco disks. The Upper Sco sources shown are those with a continuum signal-to-noise of at least 15 .

However, our modeling assumes that the dust in these disks is distributed smoothly in radius following a simple parameterization, which may not be the case. Tripathi et al. (2017) suggested that disks may be composed of optically thick substructures with a filling factor of a few tens of percent to explain an observed correlation between disk size and luminosity in 1-2 Myr old disks in Taurus and Ophiuchus. This idea is supported by theoretical models of gas and dust interactions in disks, which predict that small-scale gas pressure maxima can trap dust grains and create concentrations of optically thick continuum emission (e.g., Whipple 1972; Pinilla et al. 2012). Recent high resolution observations with ALMA have revealed a number of disks exhibiting such substructure (ALMA Partnership et al. 2015; Andrews et al. 2016; Cieza et al. 2016; Isella et al. 2016; Pérez et al. 2016; Zhang et al. 2016; Loomis et al. 2017). If young disks such as those in Lupus and Taurus are in general composed of optically thick substructures with filling factors less than 1, appearing optically thin to lower resolution observations, this could provide a way to hide dust grains migrating from the outer disk and cause the disk to appear less massive as it decreases in size. As long as the filling factor of the substructure does not increase, lower resolution observations such as those presented here and in Tazzari et al. (2017) would not detect the increase in dust surface density.

Figure 7 compares the effective radii $\left(R_{\text {eff }}\right)$ and continuum fluxes scaled to $140 \mathrm{pc}\left(L_{\mathrm{mm}}\right)$ for Upper Sco and the young stars analyzed by Tripathi et al. (2017). The Tripathi et al. (2017) disk size-luminosity relation, $R_{\mathrm{eff}} \propto L_{\mathrm{mm}}^{0.50 \pm 0.07}$, is also shown, extrapolated to the scaled flux densities of Upper Sco. Despite the difference in age and luminosity, our disks lie approximately along this extrapolation. We therefore conclude that our data is qualitatively consistent with the Tripathi et al. (2017) disk size-luminosity relation. Thus, if the disk sizeluminosity correlation is caused by optically thick substructures, the filling factor of these substructures will be roughly the same in Upper Sco as in Taurus and Ophiuchus. Higher resolution observations of samples of disks of different ages can help to further constrain the evolution of inner disks, allowing the dust surface density and optical depth to be more precisely measured as a function of radius and possibly detecting substructure.

\section{Summary}

We have presented detailed modeling of 57 circumstellar disks in the Upper Scorpius OB Association observed with ALMA. Our sample excludes the three brightest continuum disks observed in Paper I, 2MASS J15583692-2257153, 2MASS J16042165-2130284, and 2MASS J161131341838259 , instead focusing on more typical Upper Sco disks. Power-law model fits of the dust surface density to the continuum observations yielded the radial extent of dust in these disks. Similar model fits to the CO surface brightness of the disks measured the extent of CO. Using our modeling results, we compared the spatial extents of dust and CO Upper Sco disks and calculated a range of expected $\mathrm{CO}$ fluxes, comparing these model fluxes to our observed values from Paper I. The key conclusions of this paper are as follows.

1. Of the 25 analyzed disks with a continuum signal-tonoise of at least 15 , we find a median dust outer radius of $21 \mathrm{au}$. Only two of these disks had dust outer radii larger than $50 \mathrm{au}$, with none greater than $65 \mathrm{au}$, assuming an $R^{-1}$ power-law dust surface density. While this excludes the three brightest continuum sources in our sample, which appear to be more extended, it is clear that the majority of the high signal-to-noise dust disks in Upper Sco are extremely compact.

2. Among our seven disks with well-constrained dust and $\mathrm{CO}$ outer radii, four exhibited $\mathrm{CO}$ radii significantly larger than their dust radii. Given the signal-to-noise of the continuum and $\mathrm{CO}$ data, this may simply be a result 
of higher optical depths of the CO line. More sensitive observations, especially of ${ }^{13} \mathrm{CO}$ and $\mathrm{C}^{18} \mathrm{O}$, are needed to determine whether there is a true deficit of dust in the outer regions of these disks.

3. Assuming that the $\mathrm{CO}$ and dust share the same spatial distribution, the lack of $\mathrm{CO}$ detections in most of the disks is consistent with the small disk sizes inferred from the continuum.

4. Dust disks in Upper Sco are a factor of $\sim 3$ more compact than those in Taurus, Ophiuchus, and Lupus. Assuming that the continuum emission is optically thin, the lower disk masses in Upper Sco relative to Taurus and Lupus (Paper I; Ansdell et al. 2016) appear to be primarily due to the removal of material in the outer disk. We caution, however, that a more uniform analysis between samples is needed.

5. The disks in Upper Sco fall along the same sizeluminosity correlation found by Tripathi et al. (2017). If the origin of this correlation is caused by the presence of optically thick substructures, the filling factor of such structures is similar between Upper Sco and the young disks studied by Tripathi et al. (2017).

We thank the referee and statistics editor for their useful comments, which improved this manuscript. We are grateful to the ALMA staff for their assistance in the data reduction. The National Radio Astronomy Observatory is a facility of the National Science Foundation operated under cooperative agreement by Associated Universities, Inc. This paper makes use of the following ALMA data: ADS/JAO.ALMA\#2011.0.00966.S and ADS/JAO.ALMA\#2013.1.00395.S. ALMA is a partnership of ESO (representing its member states), NSF (USA), and NINS (Japan), together with NRC (Canada) and NSC and ASIAA (Taiwan), in cooperation with the Republic of Chile. The Joint ALMA Observatory is operated by ESO, AUI/NRAO, and NAOJ. This material is based upon work supported by the National Science Foundation Graduate Research Fellowship under grant No. DGE1144469. S.A.B. acknowledges support from the NSF grant No. AST-1140063. J.M.C. acknowledges support from the National Aeronautics and Space Administration under grant No. 15XRP15_20140 issued through the Exoplanets Research Progam. A.I. acknowledges support from the NSF grant No. AST-1535809 and the National Aeronautics and Space Administration grant No. NNX15AB06G. This publication makes use of data products from the Two Micron All Sky Survey, which is a joint project of the University of Massachusetts and the Infrared Processing and Analysis Center/California Institute of Technology, funded by the National Aeronautics and Space Administration and the National Science Foundation. This publication makes use of data products from the Wide-field Infrared Survey Explorer, which is a joint project of the University of California, Los Angeles, and the Jet Propulsion Laboratory/California Institute of Technology, funded by the National Aeronautics and Space Administration. This research has made use of the NASA/IPAC Extragalactic Database (NED) which is operated by the Jet Propulsion Laboratory, California Institute of Technology, under contract with the National Aeronautics and Space Administration. This work is based [in part] on observations made with the Spitzer Space Telescope, which is operated by the Jet Propulsion Laboratory, California Institute of Technology under a contract with NASA.

\section{ORCID iDs}

Scott A. Barenfeld (1) https://orcid.org/0000-0001-5222-6851

John M. Carpenter (iD https://orcid.org/0000-0003-2251-0602

Andrea Isella (ib https://orcid.org/0000-0001-8061-2207

\section{References}

Aikawa, Y., \& Nomura, H. 2006, ApJ, 642, 1152

Alexander, R., Pascucci, I., Andrews, S., Armitage, P., \& Cieza, L. 2014, in Protostars and Planets VI, ed. H. Beuther et al. (Tucson, AZ: Univ. Arizona Press), 475

ALMA Partnership, Brogan, C. L., Pérez, L. M., et al. 2015, ApJL, 808, L3 Andrews, S. M. 2015, PASP, 127, 961

Andrews, S. M., Czekala, I., Wilner, D. J., et al. 2010a, ApJ, 710, 462

Andrews, S. M., Rosenfeld, K. A., Kraus, A. L., \& Wilner, D. J. 2013, ApJ, 771,129

Andrews, S. M., \& Williams, J. P. 2007, ApJ, 659, 705

Andrews, S. M., Wilner, D. J., Espaillat, C., et al. 2011, ApJ, 732, 42

Andrews, S. M., Wilner, D. J., Hughes, A. M., et al. 2012, ApJ, 744, 162

Andrews, S. M., Wilner, D. J., Hughes, A. M., Qi, C., \& Dullemond, C. P. 2009, ApJ, 700, 1502

Andrews, S. M., Wilner, D. J., Hughes, A. M., Qi, C., \& Dullemond, C. P. 2010b, ApJ, 723, 1241

Andrews, S. M., Wilner, D. J., Zhu, Z., et al. 2016, ApJL, 820, L40

Ansdell, M., Williams, J. P., Manara, C. F., et al. 2017, AJ, 153, 240

Ansdell, M., Williams, J. P., van der Marel, N., et al. 2016, ApJ, 828, 46

Banzatti, A., Testi, L., Isella, A., et al. 2011, A\&A, 525, A12

Barenfeld, S. A., Carpenter, J. M., Ricci, L., \& Isella, A. 2016, ApJ, 827, 142 Beckwith, S. V. W., \& Sargent, A. I. 1993, ApJ, 402, 280

Bergin, E. A., Cleeves, L. I., Gorti, U., et al. 2013, Natur, 493, 644

Birnstiel, T., \& Andrews, S. M. 2014, ApJ, 780, 153

Birnstiel, T., Dullemond, C. P., \& Brauer, F. 2010, A\&A, 513, A79

Bisschop, S. E., Fraser, H. J., Öberg, K. I., van Dishoeck, E. F., \& Schlemmer, S. 2006, A\&A, 449, 1297

Carpenter, J. M., Mamajek, E. E., Hillenbrand, L. A., \& Meyer, M. R. 2006, ApJL, 651, L49

Carpenter, J. M., Ricci, L., \& Isella, A. 2014, ApJ, 787, 42

Chapillon, E., Guilloteau, S., Dutrey, A., \& Piétu, V. 2008, A\&A, 488, 565

Chen, Y.-J., Chuang, K.-J., Muñoz Caro, G. M., et al. 2014, ApJ, 781, 15

Cieza, L. A., Casassus, S., Tobin, J., et al. 2016, Natur, 535, 258

Collings, M. P., Dever, J. W., Fraser, H. J., McCoustra, M. R. S., \& Williams, D. A. 2003, ApJ, 583, 1058

Dahm, S. E., \& Carpenter, J. M. 2009, AJ, 137, 4024

Dahm, S. E., Slesnick, C. L., \& White, R. J. 2012, ApJ, 745, 56

D’Alessio, P., Calvet, N., \& Hartmann, L. 2001, ApJ, 553, 321

Dong, R., van der Marel, N., Hashimoto, J., et al. 2017, ApJ, 836, 201

Dullemond, C. P. 2012, RADMC-3D: A multipurpose radiative transfer tool, Astrophysics Source Code Library, ascl:1202.015

Dullemond, C. P., \& Dominik, C. 2005, A\&A, 434, 971

Dutrey, A., Guilloteau, S., Duvert, G., et al. 1996, A\&A, 309, 493

Dutrey, A., Guilloteau, S., \& Simon, M. 2003, A\&A, 402, 1003

Dutrey, A., Semenov, D., Chapillon, E., et al. 2014, in Protostars and Planets VI, ed. H. Beuther et al. (Tucson, AZ: Univ. Arizona Press), 317

Eisner, J. A., Bally, J. M., Ginsburg, A., \& Sheehan, P. D. 2016, ApJ, 826, 16

Espaillat, C., Muzerolle, J., Najita, J., et al. 2014, in Protostars and Planets VI, ed. H. Beuther et al. (Tucson, AZ: Univ. Arizona Press), 497

Facchini, S., Birnstiel, T., Bruderer, S., \& van Dishoeck, E. F. 2017, A\&A, 605, A16

Fayolle, E. C., Bertin, M., Romanzin, C., et al. 2011, ApJL, 739, L36

Foreman-Mackey, D., Hogg, D. W., Lang, D., \& Goodman, J. 2013, PASP, 125,306

Gorti, U., \& Hollenbach, D. 2008, ApJ, 683, 287

Gorti, U., Hollenbach, D., \& Dullemond, C. P. 2015, ApJ, 804, 29

Guidi, G., Tazzari, M., Testi, L., et al. 2016, A\&A, 588, A112

Guilloteau, S., Dutrey, A., Piétu, V., \& Boehler, Y. 2011, A\&A, 529, A105

Hardy, A., Caceres, C., Schreiber, M. R., et al. 2015, A\&A, 583, A66

Hasegawa, T. I., \& Herbst, E. 1993, MNRAS, 263, 589

Hendler, N. P., Mulders, G. D., Pascucci, I., et al. 2017, ApJ, 841, 116

Hernández, J., Hartmann, L., Calvet, N., et al. 2008, ApJ, 686, 1195

Hogerheijde, M. R., Bekkers, D., Pinilla, P., et al. 2016, A\&A, 586, A99

Hughes, A. M., Andrews, S. M., Wilner, D. J., et al. 2010, AJ, 140, 887

Hughes, A. M., Wilner, D. J., Qi, C., \& Hogerheijde, M. R. 2008, ApJ, 678,1119

Isella, A., Carpenter, J. M., \& Sargent, A. I. 2009, ApJ, 701, 260 
Isella, A., Carpenter, J. M., \& Sargent, A. I. 2010a, ApJ, 714, 1746 Isella, A., Guidi, G., Testi, L., et al. 2016, PhRvL, 117, 251101 Isella, A., Natta, A., Wilner, D., Carpenter, J. M., \& Testi, L. 2010b, ApJ, 725, 1735 Isella, A., Testi, L., Natta, A., et al. 2007, A\&A, 469, 213

Kama, M., Bruderer, S., Carney, M., et al. 2016, A\&A, 588, A108 Kitamura, Y., Momose, M., Yokogawa, S., et al. 2002, ApJ, 581, 357

Lauer, T. R., Ajhar, E. A., Byun, Y.-I., et al. 1995, AJ, 110, 2622

Lee, N., Williams, J. P., \& Cieza, L. A. 2011, ApJ, 736, 135

Long, F., Herczeg, G. J., Pascucci, I., et al. 2017, ApJ, 844, 99

Loomis, R. A., Öberg, K. I., Andrews, S. M., \& MacGregor, M. A. 2017, ApJ, 840,23

Luhman, K. L., \& Mamajek, E. E. 2012, ApJ, 758, 31

Mathews, G. S., Pinte, C., Duchêne, G., Williams, J. P., \& Ménard, F. 2013, A\&A, 558, A66

Mathews, G. S., Williams, J. P., Ménard, F., et al. 2012, ApJ, 745, 23

Mayama, S., Hashimoto, J., Muto, T., et al. 2012, ApJL, 760, L26

McClure, M. K., Bergin, E. A., Cleeves, L. I., et al. 2016, ApJ, 831, 167

Menu, J., van Boekel, R., Henning, T., et al. 2014, A\&A, 564, A93

Miotello, A., van Dishoeck, E. F., Williams, J. P., et al. 2017, A\&A, 599, A113

Öberg, K. I. 2016, in Proc. IAU Focus Meeting 29, IAU XXIX General Assembly (Cambridge: Cambridge Univ. Press), 309

Öberg, K. I., Bottinelli, S., \& van Dishoeck, E. F. 2009a, A\&A, 494, L13

Öberg, K. I., Furuya, K., Loomis, R., et al. 2015, ApJ, 810, 112

Öberg, K. I., van Dishoeck, E. F., \& Linnartz, H. 2009b, A\&A, 496, 281

Owen, J. E., Clarke, C. J., \& Ercolano, B. 2012, MNRAS, 422, 1880

Panić, O., Hogerheijde, M. R., Wilner, D., \& Qi, C. 2009, A\&A, 501, 269

Pascucci, I., Testi, L., Herczeg, G. J., et al. 2016, ApJ, 831, 125

Pecaut, M. J., Mamajek, E. E., \& Bubar, E. J. 2012, ApJ, 746, 154

Pérez, L. M., Carpenter, J. M., Andrews, S. M., et al. 2016, Sci, 353, 1519

Pérez, L. M., Carpenter, J. M., Chandler, C. J., et al. 2012, ApJL, 760, L17

Pérez, L. M., Chandler, C. J., Isella, A., et al. 2015, ApJ, 813, 41

Piétu, V., Guilloteau, S., Di Folco, E., Dutrey, A., \& Boehler, Y. 2014, A\&A, 564, A95

Piétu, V., Guilloteau, S., \& Dutrey, A. 2005, A\&A, 443, 945

Pinilla, P., Birnstiel, T., Ricci, L., et al. 2012, A\&A, 538, A114

Pinilla, P., de Boer, J., Benisty, M., et al. 2015, A\&A, 584, L4
Pollack, J. B., Hollenbach, D., Beckwith, S., et al. 1994, ApJ, 421, 615

Pontoppidan, K. M., Salyk, C., Bergin, E. A., et al. 2014, in Protostars and Planets VI, ed. H. Beuther et al. (Tucson, AZ: Univ. Arizona Press), 363

Preibisch, T., Brown, A. G. A., Bridges, T., Guenther, E., \& Zinnecker, H. 2002, AJ, 124, 404

Qi, C., D'Alessio, P., Öberg, K. I., et al. 2011, ApJ, 740, 84

R Development Core Team 2008, R: A Language and Environment for Statistical Computing (Vienna: R Foundation for Statistical Computing), http://www.R-project.org

Salyk, C., Pontoppidan, K., Corder, S., et al. 2014, ApJ, 792, 68

Siess, L., Dufour, E., \& Forestini, M. 2000, A\&A, 358, 593

Tazzari, M., Testi, L., Ercolano, B., et al. 2016, A\&A, 588, A53

Tazzari, M., Testi, L., Natta, A., et al. 2017, A\&A, 606, A88

Testi, L., Birnstiel, T., Ricci, L., et al. 2014, in Protostars and Planets VI, ed. H. Beuther et al. (Tucson, AZ: Univ. Arizona Press), 339

Testi, L., Natta, A., Scholz, A., et al. 2016, A\&A, 593, A111

Tripathi, A., Andrews, S. M., Birnstiel, T., \& Wilner, D. J. 2017, ApJ, 845, 44

Trotta, F., Testi, L., Natta, A., Isella, A., \& Ricci, L. 2013, A\&A, 558, A64

van der Marel, N., van Dishoeck, E. F., Bruderer, S., Pérez, L., \& Isella, A. 2015, A\&A, 579, A106

van der Plas, G., Ménard, F., Ward-Duong, K., et al. 2016, ApJ, 819, 102

van Zadelhoff, G.-J., Aikawa, Y., Hogerheijde, M. R., \& van Dishoeck, E. F. 2003, A\&A, 397, 789

Visser, R., van Dishoeck, E. F., \& Black, J. H. 2009, A\&A, 503, 323

Walsh, C., Juhász, A., Meeus, G., et al. 2016, ApJ, 831, 200

Weidenschilling, S. J. 1977, MNRAS, 180, 57

Whipple, F. L. 1972, in Proc. 21st Nobel Symp., From Plasma to Planet, ed. A. Evlius (New York: Wiley), 211

Williams, J. P., \& Best, W. M. J. 2014, ApJ, 788, 59

Williams, J. P., \& Cieza, L. A. 2011, ARA\&A, 49, 67

Williams, J. P., Cieza, L. A., Andrews, S. M., et al. 2013, MNRAS, 435, 1671

Woitke, P. 2015, Euro. Phys. J. Web Conf., 102, 00011

Yu, M., Evans, N. J., II, Dodson-Robinson, S. E., Willacy, K., \& Turner, N. J. 2017, ApJ, 841, 39

Zhang, K., Bergin, E. A., Blake, G. A., et al. 2016, ApJL, 818, L16

Zhang, K., Isella, A., Carpenter, J. M., \& Blake, G. A. 2014, ApJ, 791, 42 